\title{
On the Spatial Error Propagation Characteristics of Cooperative Localization in Wireless Networks
}

\author{
Bingpeng Zhou ${ }^{\dagger}$, Qingchun Chen ${ }^{\dagger}$, Pei Xiao ${ }^{\ddagger}$ and Lian Zhao ${ }^{\star}$ \\ ${ }^{\dagger}$ Southwest Jiaotong University, Chengdu, Sichuan 610031, China \\ ${ }^{\ddagger}$ University of Surrey, Guildford, Surrey GU2 7XH, UK \\ ${ }^{\star}$ Ryerson University, Toronto, Ontario, Canada, M5B 2K3 \\ zhoubingpeng@163.com, qcchen@swjtu.edu.cn, p.xiao@surrey.ac.uk, 15zhao@ryerson.ca
}

\begin{abstract}
Cooperative localization is an important technique in wireless networks. However, there are always errors in network node localization, which will spatially propagate among network nodes when performing network localization. In this paper, we study the spatial error propagation characteristics of network localization, in terms of Fisher information. Firstly, the spatial propagation function is proposed to reveal the spatial cooperation principle of network localization. Secondly, the convergence property of spatial localization information propagation is analyzed to shed light on the performance limits of network localization through spatial information propagation. It is shown that, (1) the network localization error propagates in a way of the Ohm's law in electric circuit theory, where the measurement accuracy, node location accuracy and geometric-resolution information behave like the resistances connected in parallel or series; (11) the network location error gradually diminishes with spatial localization cooperation procedures, due to the cooperative localization information propagation; (111) the essence of spatial localization cooperation among network nodes is the spatial propagation of localization information.
\end{abstract}

Index Terms-Error propagation, Fisher information, spatial cooperation, network localization.

\section{INTRODUCTION}

$\mathbf{C}$ OOPERATIVE localization plays an important role in wireless networks [1]. It provides effective localization solutions for the location-aware services such as warehousing management, location-aware security, delay tolerant network routing [2], [3] and shopping mall navigation. It revolutionizes the way people search, locate and navigate the points of interest inside buildings [4]. The localization security is prerequisite for the localization-aware services. The location privacy might be not so secure as the service provider claimed [5]. And the privacy-preserving WiFi localization scheme can be employed to overcome the privacy issues [6].

Given network measurements, the network nodes can be calibrated with each other, with an expectation to improve their location accuracies. The node calibrated in the previous round can be used to calibrate its neighboring nodes' locations.

Copyright (c) 2015 IEEE. Personal use of this material is permitted. However, permission to use this material for any other purposes must be obtained from the IEEE by sending a request to pubs-permissions@ieee.org.

This work was supported by the NSFC (No. 61271246) and the National Basic Research Program of China (973 Program No.2012CB316100). This work was also partly supported by Royal Academy of Engineering Award (batch reference 1314-2).
Hence, we can finally observe a spatial cooperation between a node and other remote nodes, which are outside its sensing coverage. However, due to the limited localization accuracy, there always exist errors in network node locations. The location errors can also be spatially propagated among network nodes in the calibration stage. Hence, the localization error propagation (EP) will become a critical issue in the network localization. Consequently, we seek answers to the following questions in this paper.

- How do the localization errors propagate spatially within the localization network?

- What are the performance limits of network localization, given fixed size of measurements among network nodes?

Since Fisher information upper bounds on the localization accuracy [7], it can be used as an information metric to measure the localization accuracy intensity. Hence, in this paper we investigate the localization error propagation and spatial localization cooperation in terms of information propagation.

In principle, if a signal is correlated with the relative geometry between the target and reference objects, it can be used as the measurement data to determine the target location, such as the visual signal (e.g., landmark picture or video) [8], [9], acoustic signal [10], wireless radio signal [11] (e.g., time-of-arrival (TOA), received signal strength (RSS) and angle-of-arrival (AOA)), channel state information [12] and optical signal [13]. The wireless localization/tracking performance limits with different measurement modalities in different environments have been studied in previous research efforts. In [14] and [15], the fundamental limits of cooperative/noncooperative localization in wide-band wireless networks are investigated to examine the impact of multipath and non-line-of-sight transmission. In [16], the spatial localization cooperation between a node and its neighboring nodes was investigated. In [17], the Cramer-Rao lower bound is presented to benchmark the simultaneous localization and tracking error in wireless sensor networks. The localization performance analysis was presented in [18] to quantify the effects of reference location uncertainties. In [19], information coupling is studied for cooperative localization by means of Fisher information analysis. The navigation information evolution is addressed in [20] to highlight the spatial and temporal cooperation in navigation networks. The fundamental limit 
of mobile localization, especially the temporal propagation of tracking errors, is revealed in [21], where different types of wireless networks measurements and performance requirements in various scenarios are considered.

However, these state-of-the-art solutions assume localization cooperation between a node and its nearby nodes. Few previous analysis considered the localization information propagation between a node and the remote nodes. Particularly, it is the general localization/tracking issue addressed in [14]-[21], where the one-step spatial cooperation among nearby nodes is assumed. Nevertheless, the whole localization information propagation, where each node may cooperate with those remote nodes outside its direct sensing range (extensive spatial cooperation), is neglected.

In this paper, the cooperative localization error propagation within the whole network is studied, which not only unveils the spatial cooperation mechanism of network localization, but also provides insights into performance limits of network localization through extensive spatial cooperation. In addition, the analysis on spatial information propagation in this paper is applied to the TOA, AOA and RSS-based localization. The main contributions of this paper are two-fold.

- The localization cooperation principle, in the spatial field, is revealed in terms of localization information propagation. It is shown that, the network localization error propagation complies with the Ohm's Law in electric circuit theory, where the measurement accuracy, node location accuracy and geometric-resolution factor behave like the resistances connected in parallel or series.

- The convergence properties and asymptotic performance are analysed to provide the insights into the performance limits of spatial localization cooperation. Even though reference node locations are inaccurate, the localization error of each node can still be reduced statistically, thanks to the cooperative localization information propagation. It is disclosed that, the essence of localization cooperation among network nodes is the spatial propagation of the associated localization information.

The remainder of this paper is organized as follows. Section II presents the system model and problem formulation. The spatial localization information propagation (SLIP) is investigated in Section III. In Section IV, the convergence property of SLIP is analysed. The asymptotic performance analysis is presented in Section V. Simulations results are presented in Section VI. Finally, Section VII concludes the paper.

\section{SySTEM MODEL}

Prior to presenting the SLIP analysis in the next section, here we clarify the system model at first.

\section{A. Network Model}

A static wireless network is considered in this paper, as shown in Fig. 1, where $M$ network nodes are randomly and uniformly distributed inside the deployment area. Due to the unavoidable acquisition errors in their initial locations, all node locations are inaccurate. The true (but unknown) location of the $i$ th network node is denoted by a $D$-dimensional column vector $\mathbf{s}_{i}$, while the coarse location (inaccurate location with a precision $\mathbf{U}_{i}$ ) is denoted by $\boldsymbol{\mu}_{i}{ }^{1}$ Generally, the true location $\mathbf{s}_{i}$ is modeled as a Gaussian variable with the center $\boldsymbol{\mu}_{i}$ and the precision $\mathbf{U}_{i}$, namely,

$$
\mathbf{s}_{i} \sim \mathcal{N}\left(\mathbf{s}_{i} \mid \boldsymbol{\mu}_{i}, \mathbf{U}_{i}\right), \forall i=1: M,
$$

where we assume node location precision $\mathbf{U}_{i}$ is independent to others, since the measurements and location estimations of different nodes are independent from each other [22], [23]. The location uncertainty is defined as the inverse of the location precision matrix $\mathbf{U}_{i}$.

This model can subsume the case where a certain node location is completely unknown when its precision $\mathbf{U}_{i} \rightarrow \mathbf{0}$. On the other hand, there is no anchor node assumed inside the whole area, and all nodes are to be located with the cooperation of other nodes. However, when $\mathbf{U}_{i}$ is sufficiently large, node $\mathbf{s}_{i}$ is equivalent to the anchor node with precisely known location.

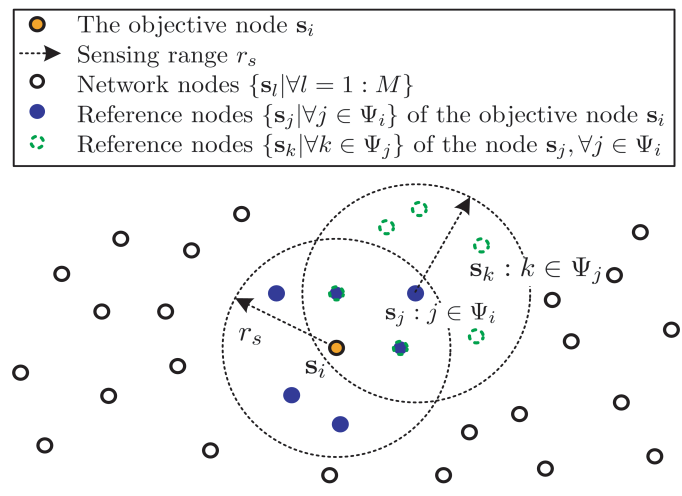

Fig. 1. Illustration of the network node deployment.

Considering the localization of node $\mathbf{s}_{i}$ (the objective node), we assume $\mathbf{s}_{i}$ is within the sensing range $r_{s}$ of $M_{i}$ nearby nodes (reference nodes), and we define the index set of these reference nodes as

$$
\Psi_{i} \doteq\left\{j:\left\|\mathbf{s}_{j}-\mathbf{s}_{i}\right\|_{2}<r_{s}, \forall j \neq i\right\},
$$

where $\|\bullet\|_{2}$ denotes the $\ell_{2}$-norm on the vector. Hence, we have that $\left|\Psi_{i}\right|=M_{i}$, where $|\bullet|$ stands for the set size. We assume these reference nodes report their coarse locations and precisions $\left\{\boldsymbol{\mu}_{j}, \mathbf{U}_{j}: \forall j \in \Psi_{i}\right\}$ to the objective node $\mathbf{s}_{i}$ to cooperatively localize it.

\section{B. Measurement Model}

The measurement model of cooperative wireless localization (incorporating the location information of nodes $\mathbf{s}_{i}$ and $\mathbf{s}_{j}$ ) is generalized as

$$
\mathrm{z}_{i, j}=h\left(\mathbf{s}_{i}, \mathbf{s}_{j}\right)+\epsilon_{i, j}, \forall j \in \Psi_{i} \text { and } \forall i=1: M,
$$

where the scalar $\mathrm{z}_{i, j}$ denotes the measurement from $\mathbf{s}_{j}$ to $\mathbf{s}_{i}$, and $\epsilon_{i, j}$ represents the measurement noise, which is generally

\footnotetext{
${ }^{1}$ The coarse location $\boldsymbol{\mu}_{i}$ and its precision matrix $\mathbf{U}_{i}$ can be derived from the previous cooperative positioning rounds, which are recorded by the node itself and will be reported to its neighbor node (the objective node to be localized) in the next positioning round.
} 
assumed to be Gaussian with zero-mean and a precision $\omega$, namely, $\epsilon_{i, j} \sim \mathcal{N}\left(\epsilon_{i, j} \mid 0, \omega\right){ }^{2}$ In particular, $h\left(\mathbf{s}_{i}, \mathbf{s}_{j}\right)$ is defined as the measurement function that depends on the distance $\left\|\mathbf{s}_{i}-\mathbf{s}_{j}\right\|_{2}$ (for the range-based methods) [21], the angle $\angle\left(\mathbf{s}_{i}, \mathbf{s}_{j}\right)$ (for the direction-based methods) [16] or the connectivity $\mathcal{C}\left(\mathbf{s}_{i}, \mathbf{s}_{j}\right)$ [27] of two nodes.

In this paper, the SLIP analysis is valid for the TOA [15], RSS [17], [28] and AOA-based localization [29], [30], where the associated measurement function $h\left(\mathbf{s}_{i}, \mathbf{s}_{j}\right)$ can be specified, respectively, as ${ }^{3}$ [21],

$$
\begin{aligned}
h_{\mathrm{TOA}}\left(\mathbf{s}_{i}, \mathbf{s}_{j}\right) & =\left\|\mathbf{s}_{i}-\mathbf{s}_{j}\right\|_{2}, \\
h_{\mathrm{RSS}}\left(\mathbf{s}_{i}, \mathbf{s}_{j}\right) & =\phi-10 \gamma \log _{10}\left\|\mathbf{s}_{i}-\mathbf{s}_{j}\right\|_{2}, \\
h_{\mathrm{AOA}}\left(\mathbf{s}_{i}, \mathbf{s}_{j}\right) & =\varphi_{j}+\frac{180}{\pi} \operatorname{actan}\left(\frac{\left[\mathbf{s}_{i}-\mathbf{s}_{j}\right]_{2}}{\left[\mathbf{s}_{i}-\mathbf{s}_{j}\right]_{1}}\right),
\end{aligned}
$$

wherein $\phi=P_{T}-L_{0}+10 \gamma \log _{10} d_{0}$ and $P_{T}$ is the transmit power, $L_{0}$ denotes the path loss associated with the reference distance $d_{0}, \gamma$ denotes the path loss exponent [31]. In addition, $[\mathbf{x}]_{k}$ stands for the $k$ th $(k=1,2)$ element of a two dimensional vector $\mathbf{x}$, and $\varphi_{j}$ stands for the direction of the antenna main lobe. Unless otherwise stated, we use $h\left(\mathbf{s}_{i}, \mathbf{s}_{j}\right)$ to denote the general range-based measurement functions.

\section{Statistical Model}

Let $\boldsymbol{c}_{i}=\operatorname{vec}\left[\mathbf{s}_{j}\right]_{\forall j \in \Psi_{i}}$ denote the vector of reference node set, where $\operatorname{vec}\left[\bullet_{j}\right]_{\forall j \in \Psi_{i}}$ yields a column vector stacked by all components $\left\{\bullet_{j}: \forall j \in \Psi_{i}\right\}$. Consider the positioning of node $\mathbf{s}_{i}$ (the objective node), and we define an $\left(M_{i}+1\right) D$ dimensional complete variable as $\boldsymbol{\alpha}_{i}:=\left[\begin{array}{c}\mathbf{s}_{i} \\ \boldsymbol{c}_{i}\end{array}\right]$. All measurements of $\mathbf{s}_{i}$ from $\boldsymbol{c}_{i}$ is stacked as $\mathbf{z}_{i}=\operatorname{vec}\left[\mathrm{z}_{i, j}\right]_{\forall j \in \Psi_{i}}$.

By assuming the measurements conditioned on $\mathbf{s}_{i}$ are mutually independent, the likelihood distribution is cast as

$$
p\left(\mathbf{z}_{i} \mid \mathbf{s}_{i}, \mathbf{s}_{j}\right)=\prod_{j \in \Psi_{i}} \frac{|\omega|^{\frac{1}{2}}}{\sqrt{2 \pi}} \exp \left(-\frac{1}{2} \omega\left(\mathrm{z}_{i, j}-h\left(\mathbf{s}_{i}, \mathbf{s}_{j}\right)\right)^{2}\right),
$$

where $|\omega|$ stands for the absolute value of precision $\omega$.

Hence, the posteriori distribution can be written as

$$
\begin{aligned}
& p\left(\boldsymbol{\alpha}_{i} \mid \mathbf{z}_{i}\right) \propto p\left(\mathbf{z}_{i} \mid \boldsymbol{\alpha}_{i}\right) p\left(\boldsymbol{\alpha}_{i}\right) \\
&=\prod_{j \in \Psi_{i}} \frac{|\omega|^{\frac{1}{2}}}{\sqrt{2 \pi}} \exp \left(-\frac{1}{2} \omega\left(\mathrm{z}_{i, j}-h\left(\mathbf{s}_{i}, \mathbf{s}_{j}\right)\right)^{2}\right) \\
& \cdot \mathcal{N}\left(\mathbf{s}_{i} \mid \boldsymbol{\mu}_{i}, \mathbf{U}_{i}\right) \mathcal{N}\left(\mathbf{s}_{j} \mid \boldsymbol{\mu}_{j}, \mathbf{U}_{j}\right),
\end{aligned}
$$

where $\propto$ implies the left term is proportional to the right.

\section{Problem Formulation}

By localization cooperation, the network nodes could improve their location accuracy. However, the priori location errors and localization errors can be propagated among network nodes. In view of this, we aim to address the following issues.

\footnotetext{
${ }^{2}$ For the TOA-based localization, we have considered the case that, the non-light-of-sight signal can be identified and removed by the identification methods [24], [25] and its positive ranging error can also be mitigated [26]. The network timer is also assumed to be synchronized.

${ }^{3}$ For the AOA-based localization (see Eq.(6)), we assume the scenario is in a 2-dimensional Euclid space, i.e., $D=2$.
}

- How does the localization information spatially propagate among inaccurate network nodes?

- Given the coarse locations as well as their precision parameters $\left\{\boldsymbol{\mu}_{i}, \mathbf{U}_{i} \mid \forall i=1: M\right\}$ of network nodes and measurements $\left\{\mathrm{z}_{i, j} \mid \forall j \in \Psi_{i}, \forall i=1: M\right\}$, what are the performance limits of node location calibration?

\section{Spatial LOCALIZATION COOPERATION}

In this section, we study the spatial localization cooperation in a perspective of localization information propagation.

\section{A. Localization Information}

In the parameter estimation theory, for an unbiased Bayesian estimation (BE) of a nondeterministic variable $\boldsymbol{\alpha}_{i}$, the covariance matrix of estimation error is lower bounded by a CramerRao lower bound (CRLB) [28] (which is denoted by $\mathcal{B}_{\mathrm{BE}}\left(\boldsymbol{\alpha}_{i}\right)$ in this paper), as follows

$$
\operatorname{cov}\left(\widehat{\boldsymbol{\alpha}}_{i}\right) \succeq \mathcal{B}_{\mathrm{BE}}\left(\boldsymbol{\alpha}_{i}\right),
$$

where the CRLB $\mathcal{B}_{\mathrm{BE}}\left(\boldsymbol{\alpha}_{i}\right)$ is calculated as the inverse of a Fisher information matrix (FIM). We define the localization accuracy (or precision) as the inverse of the error covariance matrix. The Fisher information is defined as [7]

$$
\mathcal{I}_{\mathrm{BE}}\left(\boldsymbol{\alpha}_{i}\right)=-\mathbb{E}_{\boldsymbol{\alpha}_{i}, \mathbf{z}_{i}}\left\{\nabla_{\boldsymbol{\alpha}_{i}, \boldsymbol{\alpha}_{i}^{\top}} \ln p\left(\boldsymbol{\alpha}_{i} \mid \mathbf{z}_{i}\right)\right\},
$$

where the operator $\mathbb{E}_{\boldsymbol{\alpha}_{i}, \mathbf{z}_{i}}\{\bullet\}$ denotes the expectation with respect to the distribution $p\left(\boldsymbol{\alpha}_{i}, \mathbf{z}_{i}\right)$ and $\nabla_{\boldsymbol{\alpha}_{i}, \boldsymbol{\alpha}_{i}^{\top}}$ denotes the second-order derivative.

Based on the above formulation we can see that, the FIM can be considered as the upper bound of localization accuracy. Hence, it can be used as a localization performance metric that measures the supremum of localization accuracy. In this paper, we investigate the spatial cooperation of wireless localization and the spatial propagation of localization information, in terms of Fisher information analysis.

We now calculate the full Bayesian localization information matrix of node $\mathbf{s}_{i}$ (regarded as the objective node). Suppose that the $M_{i}$ reference nodes of node $\mathbf{s}_{i}$ are successively labeled by $\mathbf{s}_{1}, \ldots, \mathbf{s}_{M_{i}}$. Assume variables $\mathbf{s}_{i}$ and $\mathbf{s}_{j}(\forall i \neq j)$ are priori independent. According to Eq. (9), its full information matrix $\mathcal{I}_{\mathrm{BE}}\left(\boldsymbol{\alpha}_{i}\right)$ can be structured as Eq. (10), where we utilize the fact that, $\mathcal{I}_{\mathrm{BE}}\left(\mathbf{s}_{i}, \mathbf{s}_{j}\right)=\mathcal{I}_{\mathrm{MLE}}\left(\mathbf{s}_{i}, \mathbf{s}_{j}\right)+\delta_{i, j} \mathcal{I}_{\mathrm{P}}\left(\mathbf{s}_{i}\right)$, while $\mathcal{I}_{\mathrm{MLE}}\left(\mathbf{s}_{i}, \mathbf{s}_{j}\right)$ and $\mathcal{I}_{\mathrm{P}}\left(\mathbf{s}_{i}\right)$ denote the maximum likelihood estimation (MLE)-based information (from the measurement only) and the priori information, respectively. Here $\delta_{i, j}=1$ if $i=j$, and zero otherwise. The generic information intensities $\mathcal{I}_{\mathrm{MLE}}\left(\mathbf{s}_{i}, \mathbf{s}_{j}\right)$ and $\mathcal{I}_{\mathrm{P}}\left(\mathbf{s}_{i}\right)$ in Eq. (10) are specified as

$$
\begin{aligned}
\mathcal{I}_{\mathrm{MLE}}\left(\mathbf{s}_{i}, \mathbf{s}_{i}\right) & =\sum_{j \in \Psi_{i}} \omega \mathbf{A}_{i, j}, \\
\mathcal{I}_{\mathrm{P}}\left(\mathbf{s}_{i}\right) & =\mathbf{U}_{i}, \\
\mathcal{I}_{\mathrm{MLE}}\left(\mathbf{s}_{i}, \mathbf{s}_{j}\right) & =-\omega \mathbf{A}_{i, j}, \forall j \in \Psi_{i}, \\
\mathcal{I}_{\mathrm{MLE}}\left(\mathbf{s}_{j}, \mathbf{s}_{j}\right) & =\omega \mathbf{A}_{i, j}, \forall j \in \Psi_{i}, \\
\mathcal{I}_{\mathrm{P}}\left(\mathbf{s}_{j}\right) & =\mathbf{U}_{j}, \forall j \in \Psi_{i}, \\
\mathcal{I}_{\mathrm{MLE}}\left(\mathbf{s}_{j}, \mathbf{s}_{k}\right) & =\mathbf{0}, \forall j \neq k, \text { and } j, k \in \Psi_{i},
\end{aligned}
$$




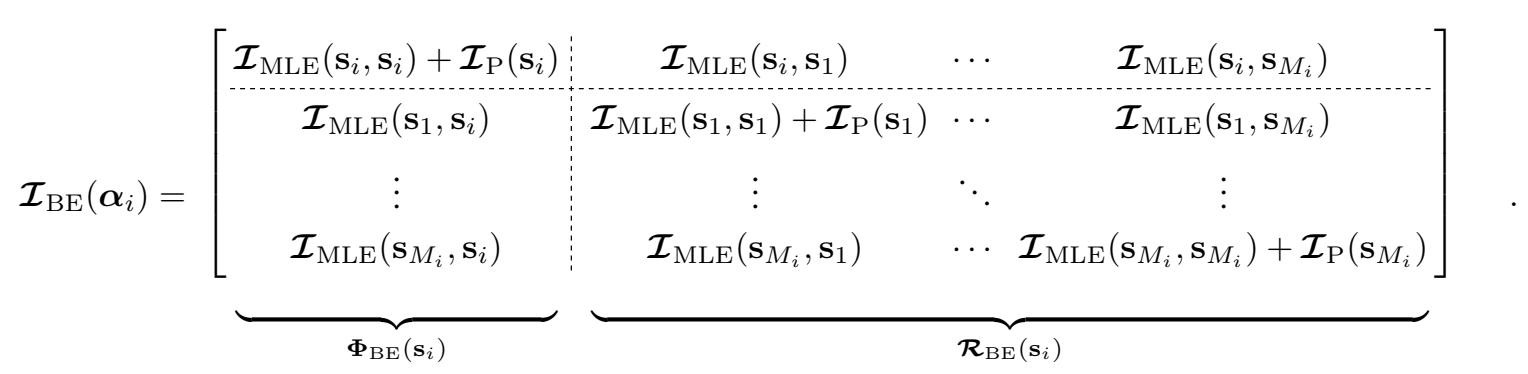

where $\mathbf{A}_{i, j}$ denotes the geometric resolution metric, ${ }^{4}$ which is given, based on three measurement methodologies, by

$$
\begin{aligned}
& \mathbf{A}_{i, j}=\mathbb{E}_{\mathbf{s}_{i}, \mathbf{s}_{j}}\left\{\nabla_{\mathbf{s}_{i}} h\left(\mathbf{s}_{i}, \mathbf{s}_{j}\right) \nabla_{\mathbf{s}_{i}^{\top}} h\left(\mathbf{s}_{i}, \mathbf{s}_{j}\right)\right\} \\
& =\left\{\begin{array}{l}
\left(\frac{10 \gamma}{\ln 10}\right)^{2} \mathbb{E}_{\mathbf{s}_{i}, \mathbf{s}_{j}}\left\{\frac{\left(\mathbf{s}_{i}-\mathbf{s}_{j}\right)\left(\mathbf{s}_{i}-\mathbf{s}_{j}\right)^{\top}}{\left\|\mathbf{s}_{i}-\mathbf{s}_{j}\right\|_{2}^{4}}\right\}, \\
\mathbb{E}_{\mathbf{s}_{i}, \mathbf{s}_{j}}\left\{\frac{\left(\mathbf{s}_{i}-\mathbf{s}_{j}\right)\left(\mathbf{s}_{i}-\mathbf{s}_{j}\right)^{\top}}{\left\|\mathbf{s}_{i}-\mathbf{s}_{j}\right\|_{2}^{2}}\right\}, \\
\left(\frac{180}{\pi}\right)^{2} \mathbb{E}_{\mathbf{s}_{i}, \mathbf{s}_{j}}\left\{\frac{\mathbf{v}_{i, j} \mathbf{v}_{i, j}^{\top}}{\left\|\mathbf{s}_{i}-\mathbf{s}_{j}\right\|_{2}^{4}}\right\},
\end{array}\right. \\
& \mathbf{v}_{i, j}=\left[\begin{array}{c}
{\left[\mathbf{s}_{j}\right]_{2}-\left[\mathbf{s}_{i}\right]_{2}} \\
{\left[\mathbf{s}_{i}\right]_{1}-\left[\mathbf{s}_{j}\right]_{1}}
\end{array}\right],(\operatorname{supposing} D=2),
\end{aligned}
$$

where $\mathbf{A}_{i, j}=\mathbf{A}_{j, i}$, and both are symmetric and have fullrank. The Bayesian information matrix of its localization is fully formulated by a $\left(\left|\Psi_{i}\right|+1\right) D$-dimensional positive semidefinite matrix $\mathcal{I}_{\mathrm{BE}}\left(\boldsymbol{\alpha}_{i}\right)$, which is calculated as Eqs. (10)-(16).

We now focus on the actual localization accuracy of node $\mathbf{s}_{i}$, given the inaccurate locations $\left\{\mathbf{s}_{j}: \forall j \in \Psi_{i}\right\}$ of all of its reference nodes. Based on the following information matrix partition (also shown in Eq. (10))

$$
\mathcal{I}_{\mathrm{BE}}\left(\mathbf{s}_{i}\right)=\left[\begin{array}{cc}
\mathcal{I}_{\mathrm{BE}}\left(\mathbf{s}_{i}, \mathbf{s}_{i}\right) & \boldsymbol{\Phi}_{\mathrm{BE}}^{\top}\left(\mathbf{s}_{i}\right) \\
\boldsymbol{\Phi}_{\mathrm{BE}}\left(\mathbf{s}_{i}\right) & \mathcal{R}_{\mathrm{BE}}\left(\mathbf{s}_{i}\right)
\end{array}\right],
$$

where $\mathcal{I}_{\mathrm{BE}}\left(\mathbf{s}_{i}, \mathbf{s}_{i}\right)=\mathcal{I}_{\mathrm{MLE}}\left(\mathbf{s}_{i}, \mathbf{s}_{i}\right)+\mathcal{I}_{\mathrm{P}}\left(\mathbf{s}_{i}\right)$, the equivalent information $\mathcal{I}_{\mathrm{EQ}}\left(\mathbf{s}_{i}\right)$ associated with $\mathbf{s}_{i}$ can be derived by using Schur's complement as

$$
\begin{aligned}
\mathcal{I}_{\mathrm{EQ}}\left(\mathbf{s}_{i}\right) & =\mathcal{I}_{\mathrm{BE}}\left(\mathbf{s}_{i}, \mathbf{s}_{i}\right)-\boldsymbol{\Phi}_{\mathrm{BE}}^{\top}\left(\mathbf{s}_{i}\right)\left(\mathcal{R}_{\mathrm{BE}}\left(\mathbf{s}_{i}\right)\right)^{-1} \boldsymbol{\Phi}_{\mathrm{BE}}\left(\mathbf{s}_{i}\right) \\
& =\sum_{j \in \Psi_{i}} \underbrace{\left(\left(\omega \mathbf{A}_{i, j}\right)^{-1}+\mathbf{U}_{j}^{-1}\right)^{-1}}_{\mathcal{H}_{i, j}}+\mathbf{U}_{i}
\end{aligned}
$$

where $\mathcal{H}_{i, j}$ is defined as the equivalent measurement information with reference node location errors. The detailed derivation can be found in APPENDIX A. The equivalent information $\mathcal{I}_{\mathrm{EQ}}\left(\mathbf{s}_{i}\right)$ retains all necessary information of localization from its full Bayesian information $\mathcal{I}_{\mathrm{BE}}\left(\boldsymbol{\alpha}_{i}\right)$ in Eq. (10), in a term of $\left[\left(\mathcal{I}_{\mathrm{BE}}\left(\boldsymbol{\alpha}_{i}\right)\right)^{-1}\right]_{[1: D, 1: D]}=\left(\mathcal{I}_{\mathrm{EQ}}\left(\mathbf{s}_{i}\right)\right)^{-1}[15]$.

We can see that, the final localization precision relies on the following information factors, i.e., the measurement precision $\omega$, the reference node location precision $\mathbf{U}_{j}$, the geometricresolution information $\mathbf{A}_{i, j}$ and the priori location precision $\mathbf{U}_{i}$. The crude measurement information (disregarding refer-

\footnotetext{
${ }^{4}$ Geometric resolution implies the capability that a localization algorithm recognizes the location difference, given certain measurement change.
}

ence node location errors) is defined as $\omega \mathbf{A}_{i, j}$. In principle, the localization performance depends on the measurement size, the density of independent reference sources, priori information, the geometric resolution of measurement methodology and the measurement noise intensity.

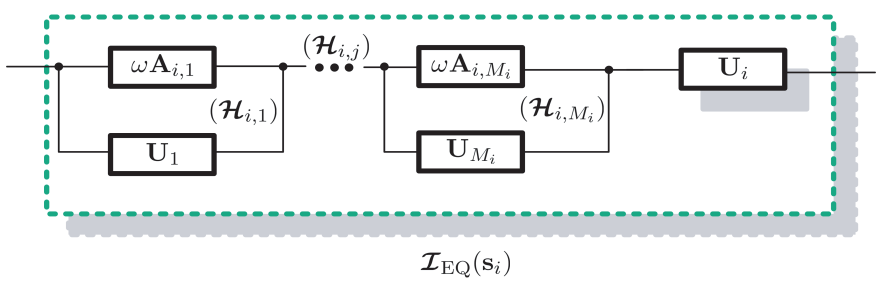

Fig. 2. Illustration of the spatial propagation of localization information.

As shown in Fig. 2, all of these localization information factors $\left(\left\{\omega \mathbf{A}_{i, j}, \mathbf{U}_{j}: \forall j \in \Psi_{i}\right\}\right.$ and $\left.\mathbf{U}_{i}\right)$ propagate like the resistances connected in serial or parallel, which complies with the Ohms Law in electric circuit theory. For the localization of node $\mathbf{s}_{i}$, the (crude) measurement information $\omega \mathbf{A}_{i, j}$ and location precision $\mathbf{U}_{j}$ of one reference node $\mathbf{s}_{j}$ can be deemed as resistances connected in parallel, forming the equivalent measurement information $\mathcal{H}_{i, j}$ (i.e., $R_{1}=\left(R_{1,1}^{-1}+R_{1,2}^{-1}\right)^{-1}$ where $R_{1}$ stands for the equivalent resistance of two parallelconnected resistances $R_{1,1}$ and $R_{1,2}$ ); these equivalent measurement information $\left\{\mathcal{H}_{i, j}: \forall j \in \Psi_{i}\right\}$ from all reference nodes and itself priori location precision $\mathbf{U}_{i}$ propagate like the resistances connected in series (resistance summation), forming the final localization information $\mathcal{I}_{\mathrm{EQ}}\left(\mathbf{s}_{i}\right)$ of $\mathbf{s}_{i}$.

\section{B. Spatial Propagation}

The localization information in Eq. (20) characterizes the initial localization accuracy of the objective node (in the first round of localization), where it has been assumed that the location accuracy of each reference node is the priori precision $\mathbf{U}_{j}, \forall j \in \Psi_{i}$. However, when all network nodes have been mutually localization more than once (here we have assumed a fixed measurement set), the location precision of its reference node is no longer the initial value $\mathbf{U}_{j}$, but the localization accuracy $\mathcal{I}_{\mathrm{EQ}}\left(\mathbf{s}_{j}\right)$ of the last round. Suppose at the $n$th round of localization, the location accuracy of its reference node $\mathbf{s}_{j}$ is denoted by $\mathcal{I}_{\mathrm{EQ}}^{(n)}\left(\mathbf{s}_{j}\right)\left(\forall j \in \Psi_{i}\right)$, then the localization information of the objective node (at the current localization round) is rewritten as

$$
\mathcal{I}_{\mathrm{EQ}}^{(n)}\left(\mathbf{s}_{i}\right)=\sum_{j \in \Psi_{i}}\left(\left(\omega \mathbf{A}_{i, j}\right)^{-1}+\left(\mathcal{I}_{\mathrm{EQ}}^{(n)}\left(\mathbf{s}_{j}\right)\right)^{-1}\right)^{-1}+\mathbf{U}_{i}
$$




$$
\mathcal{I}_{\mathrm{EQ}}^{(n)}\left(\mathbf{s}_{j}\right)=\sum_{k \in \Psi_{j} \backslash i} \underbrace{\left(\left(\omega \mathbf{A}_{j, k}\right)^{-1}+\left(\mathcal{I}_{\mathrm{EQ}}^{(n)}\left(\mathbf{s}_{k}\right)\right)^{-1}\right)^{-1}}_{\mathcal{H}_{j, k}^{(n)}}+\left(\left(\omega \mathbf{A}_{j, i}\right)^{-1}+\left(\mathcal{I}_{\mathrm{EQ}}^{(n-1)}\left(\mathbf{s}_{i}\right)\right)^{-1}\right)^{-1}+\mathbf{U}_{j}
$$

$$
\mathcal{I}_{\mathrm{EQ}}^{(n)}\left(\mathbf{s}_{i}\right)=\sum_{j \in \Psi_{i}}\left(\left(\omega \mathbf{A}_{i, j}\right)^{-1}+(\sum_{k \in \Psi_{j} \backslash i} \mathcal{H}_{j, k}^{(n)}+\underbrace{\left(\left(\omega \mathbf{A}_{j, i}\right)^{-1}+\left(\mathcal{I}_{\mathrm{EQ}}^{(n-1)}\left(\mathbf{s}_{i}\right)\right)^{-1}\right)^{-1}}_{\mathcal{H}_{j, i}^{(n-1)}}+\mathbf{U}_{j})^{-1}\right)^{-1}+\mathbf{U}_{i}
$$

where the localization information of its reference node $\mathbf{s}_{j}$ can also be similarly expressed by Eq. (21), where " $"$ " denotes set minus, and $\mathcal{H}_{j, k}^{(n)}$ denotes the equivalent measurement information from node $\mathbf{s}_{k}$ to node $\mathbf{s}_{j}$, in the $n$th localization round. By substituting Eq. (21) into Eq. (20), the localization information of $\mathbf{s}_{i}$ can be further written as Eq. (22), ${ }^{5}$ where the measurement information $\mathcal{H}_{j, k}^{(n)}$ is cast as

$$
\mathcal{H}_{j, k}^{(n)}=\left(\left(\omega \mathbf{A}_{j, k}\right)^{-1}+\left(\mathcal{I}_{\mathrm{EQ}}^{(n)}\left(\mathbf{s}_{k}\right)\right)^{-1}\right)^{-1}, \forall k \in \Psi_{j} \backslash i
$$

Eq. (22) describes a spatial propagation of the localization information among all network nodes. Prior to the discussion of its underlying mechanism, we first introduce some necessary definitions as follows.

Definition 1. (The $r$ th-order connection set $g_{r \mid i}$ ).

If $\wp_{i}\left(\mathbf{s}_{l}\right)=r$, we say the node $\mathbf{s}_{l}$ belongs to the $r$ th-order connection set of node $s_{i}$, which is defined as

$$
\boldsymbol{g}_{r \mid i}=\left\{\mathbf{s}_{l}: \wp_{i}\left(\mathbf{s}_{l}\right)=r, \forall l \neq i\right\},
$$

where $\wp_{i}\left(s_{l}\right)$ denotes the minimum hops that $\mathbf{s}_{l}$ connects (in the sense of localization observation) to the node $\mathrm{s}_{i}$.

$\wp_{i}\left(s_{l}\right)$ is also referred to as the connection order in the following. In addition, if there is no observation connections from $\mathbf{s}_{l}$ to $\mathbf{s}_{i}$, we denote $\wp_{i}\left(s_{l}\right)=\infty$. In view of this, the reference nodes in $\Psi_{i}$ of the objective node $\mathbf{s}_{i}$ is equivalent to its firstorder connection set $\boldsymbol{g}_{1 \mid i}$. An example of connection tree with respect to the objective node $\mathbf{s}_{i}$, according to the connection orders of network nodes, is shown in Fig. 3. All nodes can be classified accordingly as $\boldsymbol{g}_{1 \mid i}, \boldsymbol{g}_{2 \mid i}, \cdots$. Considering the case of whole network, a connection graph can be finally figured out, and the reference cluster size $\left|\Psi_{i}\right|$ of a generic node $\mathbf{s}_{i}$ can also be read as its connection multiplicity.

Theorem 1. A node $s_{l}$ can contribute to the localization of another node $s_{i}$ through spatial cooperation, only if its connection order $\wp_{i}\left(s_{l}\right)<\infty$ holds, namely there exists a connection link from $\mathbf{s}_{l}$ to $\mathrm{s}_{i}$.

Proof: The reasonableness of Theorem 1 lies in the localization information propagation equation (22). If $\wp_{i}\left(s_{l}\right)<\infty$, then there must be a connection link to make the equivalent measurement information $\mathcal{H}_{j, k}^{(n)}$ of the 2nd-order connection nodes to be not lower than zero, namely $\mathcal{H}_{j, k}^{(n)} \succeq \mathbf{0}, \forall j \in \Psi_{i}$. Hence, its localization accuracy information $\mathcal{I}_{\mathrm{EQ}}^{(n)}\left(\mathbf{s}_{l}\right)$ can finally propagate to the objective node $\mathbf{s}_{i}$, thus to improve

\footnotetext{
${ }^{5} \mathrm{We}$ suppose that there is no measurement of $\mathbf{s}_{k}$ from $\mathbf{s}_{i}$, namely $i \notin \Psi_{k}$
} where $k \in \Psi_{j}$ and $j \in \Psi_{i}$. In other words, $k \notin \Psi_{i} \bigcap \Psi_{j}$.

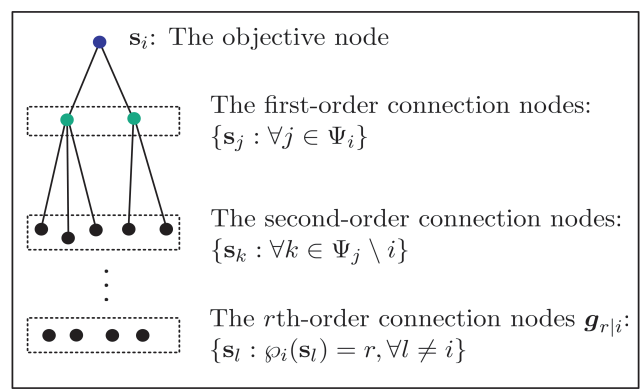

Fig. 3. Illustration of network nodes classification according to its connection order $\wp_{i}$ to the objective node $\mathbf{s}_{i}$.

the localization accuracy $\mathcal{I}_{\mathrm{EQ}}^{(n)}\left(\mathbf{s}_{i}\right)$ of node $\mathbf{s}_{i}$.

The remote node $\mathbf{s}_{k}$ can also contribute to the localization of node $\mathbf{s}_{i}$ through the spatial propagation of localization information $\mathcal{I}_{\mathrm{BE}}^{(n)}\left(\mathbf{s}_{k}\right) \rightarrow \mathcal{I}_{\mathrm{BE}}^{(n)}\left(\mathbf{s}_{j}\right) \rightarrow \mathcal{I}_{\mathrm{BE}}^{(n)}\left(\mathbf{s}_{i}\right),{ }^{6}$ even though it is out of the sensing area of the objective node $\mathbf{s}_{i}$ (namely $k \notin \Psi_{i}$ ). A simple case of connection network is shown in

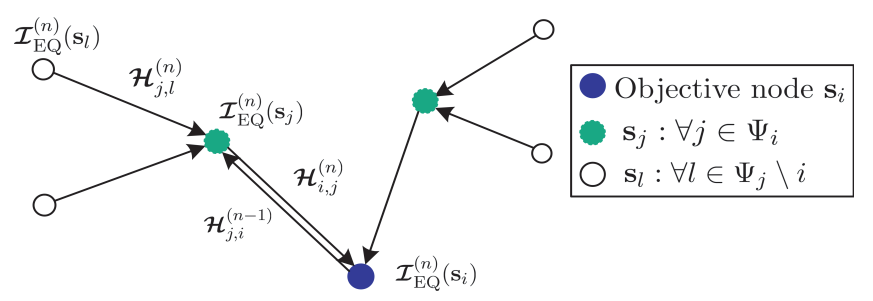

Fig. 4. Illustration of spatial propagation of localization information from nodes with various connection orders to the objective node $\mathbf{s}_{i}$.

Fig. 4, where the spatial localization information propagates according to Eq. (22). By passing the equivalent measurement information $\mathcal{H}_{j, k}^{(n)}$ through those nodes with each connection order, the objective node $\mathbf{s}_{i}$ incorporates the localization information $\mathcal{I}_{\mathrm{EQ}}^{(n)}\left(\mathbf{s}_{j}\right)$ and $\mathcal{I}_{\mathrm{EQ}}^{(n)}\left(\mathbf{s}_{k}\right), \forall j, k \neq i$, and the last localization information $\mathcal{I}_{\mathrm{EQ}}^{(n-1)}\left(\mathbf{s}_{i}\right)$ of its own. As shown in Eq. (22), the term $\mathcal{H}_{j, k}\left(\forall j \in \Psi_{i}\right.$ and $\left.\forall k \in \Psi_{j} \backslash i\right)$ opens a gate that allows the localization information from the remote nodes $\boldsymbol{g}_{r \mid i}(\forall r \geq 2)$ to come into $\mathcal{I}_{\mathrm{EQ}}\left(\mathbf{s}_{i}\right)$. Hence, the localization contribution of a remote node $\mathbf{s}_{l}$ to $\mathbf{s}_{i}$ depends on its equivalent measurement information $\mathcal{H}_{l, m}, \forall m \in \Psi_{l}$, and its connection order $\wp_{i}\left(\mathbf{s}_{l}\right)$ to $\mathbf{s}_{i}$. In addition, the localization accuracy of $\mathbf{s}_{i}$ depends on its connection multiplicity $\left|\Psi_{i}\right|$.

${ }^{6}$ Here, the symbol " $\rightarrow$ " denotes the direction of localization information propagation, rather than the asymptotic process under mathematical limits. 


\section{Convergence Analysis}

In this section, we will analyze SLIP convergence, which will shed light on the network localization performance limits.

\section{A. SLIP Convergence}

Theorem 2. Given the information $\left\{\boldsymbol{\mu}_{i}, \mathbf{U}_{i}: \forall i=1: M\right\}$ of all node locations and measurements $\left\{\mathrm{z}_{i, j}: \forall i, j\right\}$, each node location accuracy $\mathcal{I}_{\mathrm{EQ}}\left(\mathrm{s}_{i}\right)$ can converge to an upper state $\mathcal{I}_{\mathrm{EQ}}^{\star}\left(\mathrm{s}_{i}\right)$ through spatial localization cooperation. All node location accuracy $\left\{\mathcal{I}_{\mathrm{EQ}}^{\star}\left(\mathbf{s}_{i}\right): \forall i\right\}$ will reach a balance state until there are additional measurements.

Proof: The associated proof is given in APPENDIX B.

The balance state of localization information propagation represents the final accuracy of node localization. Theorem 2 tells us that, even though all node locations are not accurate (which means the reference node locations are also inaccurate in the context of cooperative network calibration), given network measurements, each node location accuracy can be improved statistically, through spatial localization cooperation. Namely, there always exists valuable localization information to be exploited even for an inaccurate reference node.

In the following, we aim at analyzing its balancing process through spatial cooperation and finding out its balance state. We can see from Eq. (22) that, due to the presence of $\mathcal{H}_{j, k}$, $\forall j \in \Psi_{i}$ and $\forall k \in \Psi_{j} \backslash i$, the localization information of its remote nodes $\boldsymbol{g}_{r \mid i},\left(\forall r \in \mathbb{N}_{+}\right)$can determine the balance state $\mathcal{I}_{\mathrm{EQ}}\left(\mathbf{s}_{i}\right)$. The SLIP balance state of any node is jointly determined by $\mathcal{H}_{j, k}$ of other connecting nodes. Let $\mathcal{I}_{\mathrm{EQ}}^{\star}\left(\mathbf{s}_{i}\right)$ denote the SLIP balance state of node $\mathbf{s}_{i}, \forall i=1: M$, then the balance states of all nodes are the solutions to the joint balance equations below,

$$
\left\{\begin{aligned}
\mathcal{I}_{\mathrm{EQ}}^{\star}\left(\mathbf{s}_{i}\right) & =\sum_{j \in \Psi_{i}} \mathcal{H}_{i, j}^{\star}+\mathbf{U}_{i}, \\
\mathcal{I}_{\mathrm{EQ}}^{\star}\left(\mathbf{s}_{j}\right) & =\sum_{k \in \Psi_{j}} \mathcal{H}_{j, k}^{\star}+\mathbf{U}_{j}, \\
\vdots & \text { (for all of the rest nodes) }
\end{aligned}\right.
$$

where $\mathcal{H}_{i, j}^{\star}$ denotes the equivalent measurement information with respect to the balanced localization information $\mathcal{I}_{\mathrm{EQ}}^{\star}\left(\mathbf{s}_{j}\right)$ of $\mathbf{s}_{j}, \forall j \in \Psi_{i}$, which is expressed as

$$
\mathcal{H}_{i, j}^{\star}=\left(\left(\omega \mathbf{A}_{i, j}\right)^{-1}+\left(\mathcal{I}_{\mathrm{EQ}}^{\star}\left(\mathbf{s}_{j}\right)\right)^{-1}\right)^{-1},
$$

and so is $\mathcal{H}_{j, k}^{\star}$. The corresponding localization information gain from spatial propagation is defined as

$$
\begin{aligned}
\mathcal{G}\left(\mathbf{s}_{i}\right) & \doteq \mathcal{I}_{\mathrm{EQ}}^{\star}\left(\mathbf{s}_{i}\right)-\left.\mathcal{I}_{\mathrm{EQ}}^{(n)}\left(\mathbf{s}_{i}\right)\right|_{n=1} \\
& =\mathcal{I}_{\mathrm{EQ}}^{\star}\left(\mathbf{s}_{i}\right)-\sum_{j \in \Psi_{i}} \mathcal{H}_{i, j}-\mathbf{U}_{i},
\end{aligned}
$$

where $\mathcal{H}_{i, j}$ without considering spatial information propagation is given by Eq. (20). The information gain comes from the spatial cooperation between $\mathbf{s}_{i}$ with its various order of connection node sets $\boldsymbol{g}_{r \mid i}, \forall r \in \mathbb{N}_{+}$.

The above analysis unveils the potential localization information inherent in network nodes connected mutually, which can improve the localization accuracy further. It is disclosed that, the essence of spatial localization cooperation is just the spatial propagation of localization information.

Note that, the number of balance equations in Eq. (25) equals to the number of nodes inside the localization network, and their balance equations are coupled with each other. The SLIP balance states of all nodes depend on not only the node connection graph but also their own equivalent measurement information and their own priori location information. Given a network with $M$ nodes, the number of node connection graphes is on the order of $\frac{(M-1)^{M}}{M !}$. Hence, the closed-form solution to Eq. (25) is intractable due to the large amount of node connection situations. However, a numeric solution based on the iteration of SLIP functions in Eq. (22) $(\forall i=1: M)$ is feasible. By assuming some regular properties, the SLIP analysis significantly reduces the complexity and exploits the spatial cooperation among the nodes.

\section{B. Generic Solution}

Since the amount of node connection situations is nearly exponential-growing with the number of nodes, we study a generic network case to derive the SLIP balance state, where some regular properties are assumed as follows.

- Assume $\mathbb{E}_{\mathbf{s}_{i}}\left\{\mathbf{U}_{i}\right\}=\mathbf{U}, \forall i=1: M$.

- Assume $\mathbb{E}_{\mathbf{s}_{i}, \mathbf{s}_{j}}\left\{\omega \mathbf{A}_{i, j}\right\}=\boldsymbol{\Lambda}, \forall j \in \Psi_{i}, \forall i=1: M$.

- Assume $\left|\Psi_{i}\right|=\Phi, \forall i=1: M$.

- Assume $\mathbb{E}_{\mathbf{s}_{i}}\left\{\mathcal{I}_{\mathrm{BE}}^{\star}\left(\mathbf{s}_{i}\right)\right\}=\boldsymbol{J}_{\star}, \forall i=1: M$.

These four items indicate identical properties for all nodes, which means that, from the perspective of long-term statistical averaging, $\mathbf{U}_{i}, \mathbf{A}_{i, j}$ and connection multiplicity $\left|\Psi_{i}\right|$ of network node is identical to each other. That is to say, there is no special configuration for any node. On this basis, the associated balance equation is reformed as

$$
\boldsymbol{J}_{\star}=\Phi\left(\boldsymbol{\Lambda}^{-1}+\boldsymbol{J}_{\star}^{-1}\right)^{-1}+\mathbf{U},
$$

which can be further expressed as

$$
\boldsymbol{J}_{\star} \boldsymbol{\Lambda}^{-1} \boldsymbol{J}_{\star}-\mathbf{U G} \boldsymbol{J}_{\star}-\mathbf{U}=\mathbf{0},
$$

where the constructed matrix $\mathbf{G}$ is given by

$$
\mathbf{G}=(\Phi-1) \mathbf{U}^{-1}+\boldsymbol{\Lambda}^{-1} .
$$

The derivation can be found in APPENDIX C.

Then its balance state is obtained as

$$
\boldsymbol{J}_{\star}=\frac{1}{2} \boldsymbol{\Lambda}^{\frac{1}{2}}\left(\boldsymbol{\Lambda}^{-\frac{1}{2}}(4 \mathbf{U}+\mathbf{U G} \boldsymbol{\Lambda} \mathbf{G U}) \boldsymbol{\Lambda}^{-\frac{1}{2}}\right)^{\frac{1}{2}} \boldsymbol{\Lambda}^{\frac{1}{2}}+\frac{1}{2} \mathbf{U G} \boldsymbol{\Lambda} .
$$

The derivation of Eq. (31) is detailed in APPENDIX D.

Under such generic assumptions, given the averaged priori precision $\mathbf{U}$ and the averaged equivalent measurement information $\boldsymbol{\Lambda}$, the generic balance state $\boldsymbol{J}_{\star}$ mainly depends on the average connection multiplicity $\Phi$ of each node. Moreover, in such a generic network, the localization information gain from spatial propagation is specified as

$$
\begin{aligned}
\mathcal{G}= & \frac{1}{2} \boldsymbol{\Lambda}^{\frac{1}{2}}\left(\boldsymbol{\Lambda}^{-\frac{1}{2}}(4 \mathbf{U}+\mathbf{U G} \boldsymbol{\Lambda} \mathbf{G} \mathbf{U}) \boldsymbol{\Lambda}^{-\frac{1}{2}}\right)^{\frac{1}{2}} \boldsymbol{\Lambda}^{\frac{1}{2}} \\
& -\Phi \mathbf{U}(\boldsymbol{\Lambda}+\mathbf{U})^{-1} \boldsymbol{\Lambda}+\frac{1}{2}(\Phi \boldsymbol{\Lambda}-\boldsymbol{\Lambda}-\mathbf{U}) .
\end{aligned}
$$




\section{ASYMPTOTIC ANALYSIS}

In this section we aim at analyzing the asymptotic properties of spatial propagation of localization information to investigate its performance limits.

Theorem 3. The final localization accuracy of each node is upper and lower bounded as

$$
\mathbf{U}_{i} \preceq \mathcal{I}_{\mathrm{EQ}}^{\star}\left(\mathbf{s}_{i}\right) \preceq \Theta,
$$

where the upper bound is defined as

$$
\boldsymbol{\Theta}=\sum_{j \in \Psi_{i}} \omega \mathbf{A}_{i, j}+\mathbf{U}_{i} .
$$

Proof: When the localization accuracy of all reference nodes of a generic node $\mathbf{s}_{i}$ is sufficiently large or arbitrarily small, based on the propagation function in Eq. (21), the localization accuracy of $\mathbf{s}_{i}$ becomes, respectively,

$$
\begin{aligned}
& \lim _{\substack{\mathcal{I}_{\mathrm{EQ}}\left(\mathbf{s}_{j}\right) \rightarrow \infty \\
\forall j \in \Psi_{i}}} \mathcal{I}_{\mathrm{EQ}}\left(\mathbf{s}_{i}\right)=\sum_{j \in \Psi_{i}} \omega \mathbf{A}_{i, j}+\mathbf{U}_{i} \doteq \boldsymbol{\Theta}, \\
& \lim _{\substack{\mathcal{I}_{\mathrm{EQ}}\left(\mathbf{s}_{j}\right) \rightarrow \mathbf{0} \\
\forall j \in \Psi_{i}}} \mathcal{I}_{\mathrm{EQ}}\left(\mathbf{s}_{i}\right)=\mathbf{U}_{i},
\end{aligned}
$$

where $\mathcal{I}_{\mathrm{EQ}}\left(\mathbf{s}_{j}\right) \rightarrow \infty$ implies $\mathcal{I}_{\mathrm{EQ}}\left(\mathbf{s}_{j}\right)-\mathbf{N} \succeq \mathbf{0}, \forall \mathbf{N}$ with $D$-dimensions.

Due to the nondecreasing property of $\mathcal{I}_{\mathrm{EQ}}\left(\mathbf{s}_{i}\right)$ with respect to its reference node location accuracy $\mathcal{I}_{\mathrm{EQ}}\left(\mathbf{s}_{j}\right)$, the localization accuracy of $\mathbf{s}_{i}$ is bounded as

$$
\mathbf{U}_{i} \preceq \mathcal{I}_{\mathrm{EQ}}\left(\mathbf{s}_{i}\right) \preceq \boldsymbol{\Theta} .
$$

Since the balance state $\mathcal{I}_{\mathrm{BE}}^{\star}\left(\mathbf{s}_{i}\right)$ is a specific value inside the range area of localization information $\mathcal{I}_{\mathrm{EQ}}\left(\mathbf{s}_{i}\right)$, thus $\mathcal{I}_{\mathrm{EQ}}^{\star}\left(\mathbf{s}_{i}\right)$ is also bounded by $\mathbf{U}_{i}$ and $\boldsymbol{\Theta}$, as shown in Eq. (33).

Theorem 3 implies that, the spatial localization cooperation gain $\mathcal{G}\left(\mathbf{s}_{i}\right)$ defined in Eqs. (27) and (30) is not more than $\boldsymbol{\Theta}-\mathbf{U}_{i}=\sum_{j \in \Psi_{i}} \omega \mathbf{A}_{i, j}$.

We now focus on analyzing the asymptotic performance of generic network localization introduced in Section V-B.

Theorem 4 . The balance state $J_{\star}$ of network localization accuracy is asymptotically linear with the average multiplicity $\Phi$ of network node connection, and the growth rate of final localization information $J_{\star}$ with respect to node number is the averaged measurement information $\Lambda$.

Proof: Based on Eq. (30), two involved items in Eq. (31) can be further expanded as follows.

$$
\begin{aligned}
\mathbf{U G} \boldsymbol{\Lambda} \mathbf{G} \mathbf{U} & =(\Phi-1)^{2} \boldsymbol{\Lambda}+2(\Phi-1) \mathbf{U}+\mathbf{U} \boldsymbol{\Lambda}^{-1} \mathbf{U}, \\
\mathbf{U G} \boldsymbol{\Lambda} & =(\Phi-1) \boldsymbol{\Lambda}+\mathbf{U} .
\end{aligned}
$$

Consequently, the balance state is rewritten as Eq. (40). In addition, $\Phi^{-1} \boldsymbol{J}_{\star}$ reflects the equivalent information contributed from each connection node. When the averaged connection multiplicity of each node becomes very large, we have

$$
\lim _{\Phi \rightarrow \infty} \Phi^{-1} \boldsymbol{J}_{\star}=\boldsymbol{\Lambda} .
$$

Thus Theorem 4 is proved.

Theorem 4 implies that, in a dense network case where all nodes are strongly connected to each other, the equivalent localization information contributed from one connected node is almost the averaged measurement information $\boldsymbol{\Lambda}$. Hence, the final localization accuracy of a generic node is in a level of $\Phi \boldsymbol{\Lambda}$, in cooperation with $\Phi$ connected nodes. In other words, whenever a new reference node is added for each node, there will be localization performance gain of $\boldsymbol{\Lambda}$.

Theorem 5. When the measurement precision $\omega$ is sufficiently large, we have

$$
\lim _{\omega \rightarrow \infty} \mathcal{I}_{\mathrm{EQ}}\left(\mathbf{s}_{i}\right)=\left(\sum_{j \in \Psi_{i}} \mathbf{U}_{j}+\mathbf{U}_{i}\right)^{-1} .
$$

Theorem 5 indicates that, when a sufficiently large size of measurements are sampled such that the measurement error is arbitrarily small, the localization accuracy will depend on the priori precision factors only, which is independent to the geometric resolution and measurement modalities.

\section{Numerical Results}

We now present extensive simulation results to validate the spatial propagation analysis in this paper.

\section{A. Simulation Setting}

In order to configure the priori location precision of network node in the simulations, we assume it complies with a Wishart distribution, namely, $\mathbf{U}_{i} \sim \mathcal{W}\left(\mathbf{U}_{i} \mid \mathbf{V}, \wp\right), \forall i=1: M$, where $\mathbf{V}$ denotes the scaling matrix and $\wp$ stands for the associated degree of freedom. The reason of employing Wishart distribution lies in the facts that, it is commonly used to model the precision parameter of a Gaussian distribution, and it is also the conjugate priori of the Gaussian distribution precision [32]. Consequently, we can see that, the averaged priori precision of the network node locations is $\wp \mathbf{V}$, which can reflect the level of location uncertainties of network nodes. We use the matrix trace as the metric to assess the localization accuracy or error, since we consider the fact that, it is the trace of equivalent Cramer-Rao lower bound that acts upon the mean squared localization errors, namely, $\operatorname{tr}\left(\boldsymbol{\mathcal { B }}_{\mathrm{EQ}}\left(\mathbf{s}_{i}\right)\right) \leq \operatorname{cov}\left(\widehat{\mathbf{s}}_{i}\right)$ where we define $\mathcal{B}_{\mathrm{EQ}}\left(\mathbf{s}_{i}\right)=\left(\mathcal{I}_{\mathrm{EQ}}\left(\mathbf{s}_{i}\right)\right)^{-1}$. All results are averaged over a total of 1000 simulation runs.

TABLE I

Simulation SETtings

\begin{tabular}{ccccc}
\hline & $M$ & $\mathbf{V}$ & $\wp$ & $\omega$ \\
\hline $\mathrm{A}$ & 300 & $1 / 500: 1 / 10 \mathbf{I}$ & 10 & 1 \\
$\mathrm{~B}$ & 300 & $1 / 100 \mathbf{I}$ & 10 & $1 / 7: 1$ \\
$\mathrm{C}$ & $100: 300$ & $1 / 100 \mathbf{I}$ & 10 & 1 \\
$\mathrm{D}$ & 300 & $1 / 100 \mathbf{I}$ & 10 & 1 \\
\hline
\end{tabular}

In this section, we consider the RSS-based network localization in an area of $100[\mathrm{~m}] \times 100[\mathrm{~m}]$. We also assume that, $\gamma=3, P_{T}=50, L_{0}=1, d_{0}=1$ and $r_{s}=20[\mathrm{~m}]$ throughout the simulations. In order to clearly demonstrate the spatial propagation behaviour of localization information (or errors) in different environments, we first simulate Scenarios A, B and $C$ in this section. The simulation settings are summarized in Table I. Furthermore, Scenario D is simulated to examine the details of spatial propagation. 


$$
\boldsymbol{J}_{\star}=\frac{\Phi-1}{2} \boldsymbol{\Lambda}^{\frac{1}{2}}\left(\boldsymbol{\Lambda}^{-\frac{1}{2}}\left(\boldsymbol{\Lambda}+\frac{2(\Phi+1) \mathbf{U}}{(\Phi-1)^{2}}+\frac{\mathbf{U} \boldsymbol{\Lambda}^{-1} \mathbf{U}}{(\Phi-1)^{2}}\right) \boldsymbol{\Lambda}^{-\frac{1}{2}}\right)^{\frac{1}{2}} \boldsymbol{\Lambda}^{\frac{1}{2}}+\frac{(\Phi-1) \boldsymbol{\Lambda}}{2}+\frac{\mathbf{U}}{2} .
$$

\section{B. Simulation Results}

The convergence behavior of spatial propagation of the localization information in different environments (i.e., scenarios A, B and C) are shown in Fig. 5, and its localization error propagation convergence is correspondingly shown in Fig. 6, wherein those three subfigures correspond to Scenarios A, B and $\mathrm{C}$, respectively.

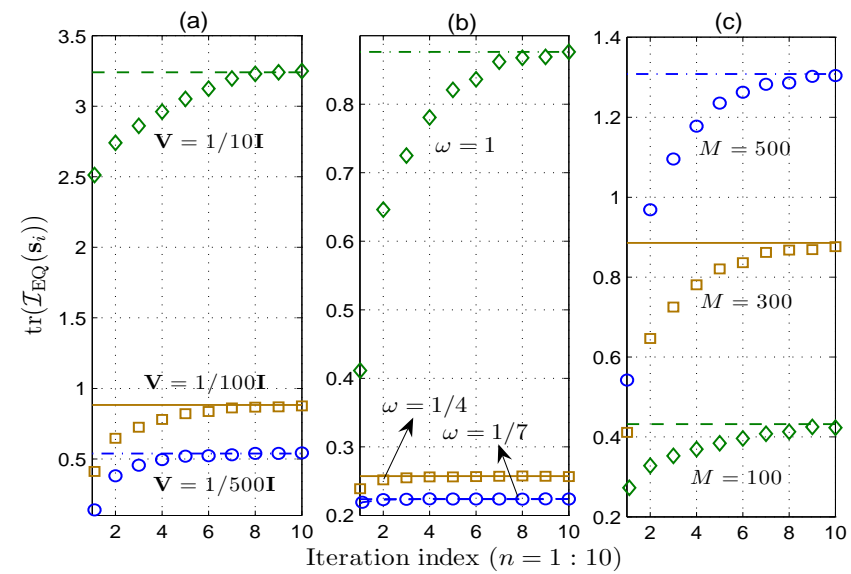

Fig. 5. Spatial propagation convergence of the localization information. The discontinuous curves stand for the localization information $\mathcal{I}_{\mathrm{EQ}}^{(n)}\left(\mathbf{s}_{i}\right)$, while the horizontal lines correspond to the associated balance states $\mathcal{I}_{\mathrm{EQ}}^{\star}\left(\mathbf{s}_{i}\right)$. In particular, at the first round of localization (namely $n=1$ ), $\mathcal{I}_{\mathrm{EQ}}\left(\mathbf{s}_{i}\right)$ corresponds to the localization information without spatial information propagation, wherein the location information that each reference node $\mathbf{s}_{j}\left(\forall j \in \Psi_{i}\right)$ propagates to the objective node $\mathbf{s}_{i}$ is its original priori $\mathbf{U}_{j}$ only. However, from the iterations of $n \geq 2$, the cooperative localization begins to benefit from the spatial propagation of localization information among network nodes. Gradually, the network localization information converges to a higher level and keep balance, as unveiled in Theorem 2.
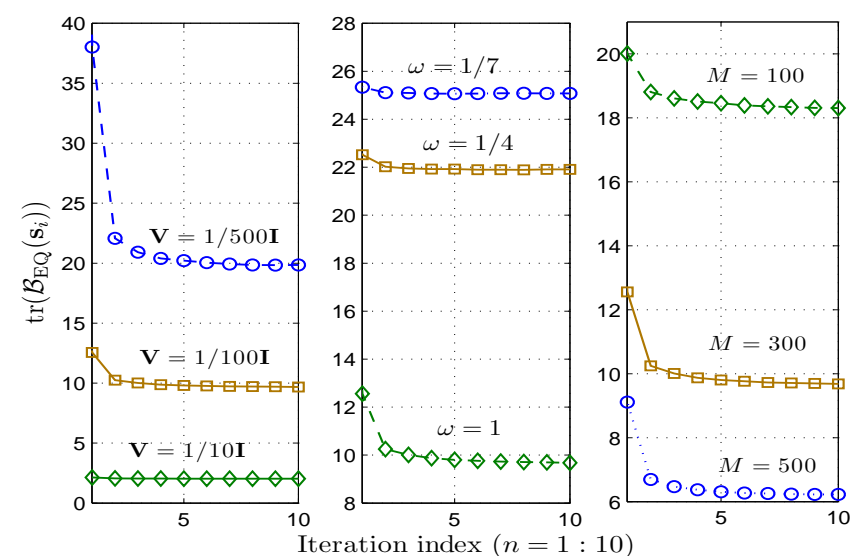

Fig. 6. Spatial propagation convergence of the localization errors.

As shown in Fig. 5 and Fig. 6, the localization of all network nodes benefits from the spatial propagation of corresponding localization information (see more details in Fig. 7). Through spatial cooperation, all node location precision approaches up to the associated balance state. In addition, in terms of mean squared localization errors, the network localization with less priori location information and larger measurement information benefits more from the spatial cooperation, as shown in Fig. 6.

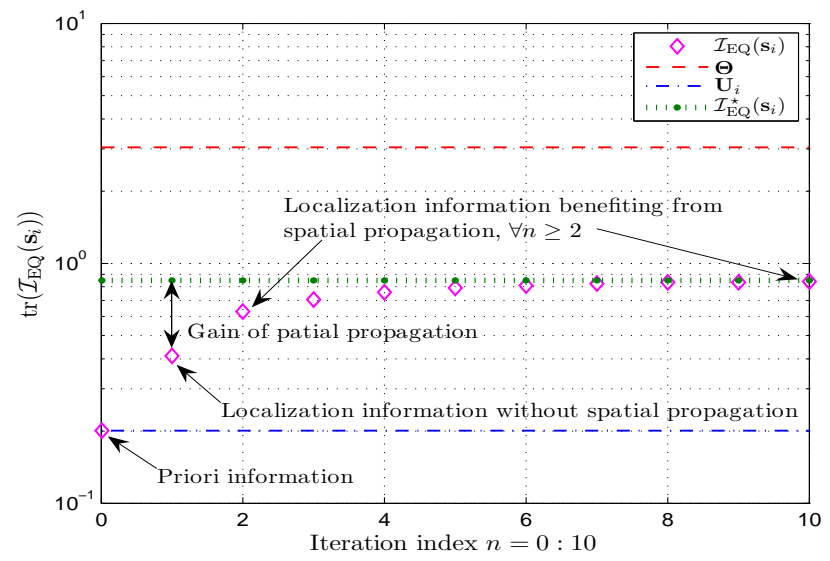

Fig. 7. Information gain inside spatial localization propagation.

We examine the localization performance bounds (see Theorem 3) and the localization performance gain over scenario D in Table I. As shown in Fig. 7, the localization information $\mathcal{I}_{\mathrm{EQ}}\left(\mathbf{s}_{i}\right)$ (as well as its balance state $\mathcal{I}_{\mathrm{EQ}}^{\star}\left(\mathbf{s}_{i}\right)$ ) is upper and lower bounded by $\Theta$ and $\mathbf{U}_{i}$, respectively. However, due to the limited final localization accuracy $\mathcal{I}_{\mathrm{EQ}}^{\star}\left(\mathbf{s}_{j}\right)$ of reference node $\mathbf{s}_{j}\left(\forall j \in \Psi_{i}\right)$, there still exists a gap between $\mathcal{I}_{\mathrm{EQ}}^{\star}\left(\mathbf{s}_{i}\right)$ with its upper bound $\Theta$.

Moreover, as shown in Fig. 7, the localization information at the first round of propagation (i.e., $n=1$ ) denotes the performance of traditional cooperative localization schemes, where there is localization cooperation only between a node and its nearby nodes. While when $n \geq 2$, the localization information of a node can be further leveled up due to the spatial cooperation with its remote nodes. Hence, benefiting from spatial propagation, the network localization reap more localization cooperation gain $\mathcal{G}\left(\mathbf{s}_{i}\right)$ from its various order of connection node set $\boldsymbol{g}_{r \mid i}, \forall r \in \mathbb{N}_{+}$.

Figs. 8(a) and 8(b) present the balanced localization information $\boldsymbol{J}_{\star}$ (in a generic case considered in Section IV-B) and its growth rate $\Omega$ in different environments, where we set $\mathbf{V}=1 / 100 \mathbf{I}$ and $\wp=10$, while the measurement precision $\omega$ varies in $[1 / 7,1]$. In particular, the averaged connection multiplicity $\Phi$ of each node is set to rang from 1 to 49 to unveil its localization information. We can see from Fig. 8(a) that, $\boldsymbol{J}_{\star}(\Phi)$ is asymptotically linear with the connection multiplicity $\Phi$, as unveiled in Theorem 4. This conclusion can also be observed from Fig. 8(b), where the corresponding growth rate 

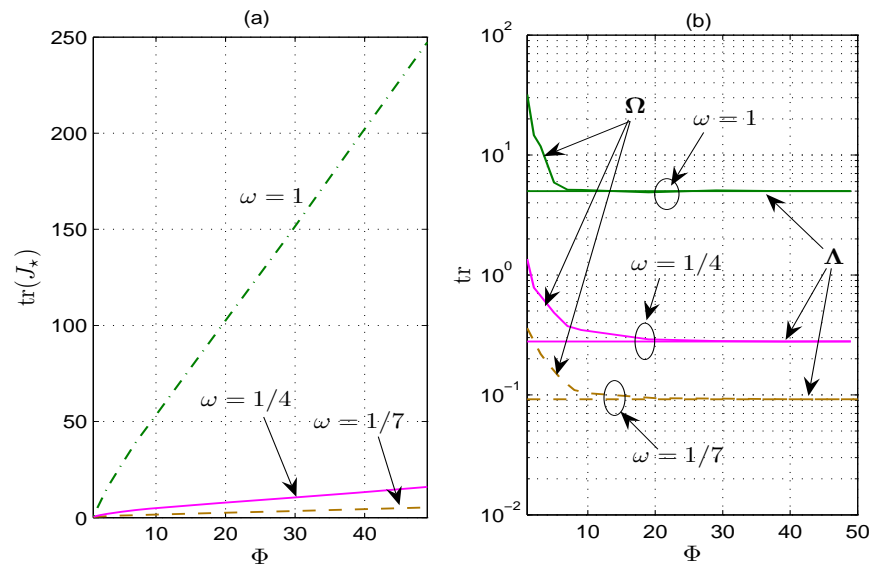

Fig. 8. Balanced localization information $\boldsymbol{J}_{\star}$ and its growth rate $\boldsymbol{\Omega}$ under different connection multiplicities $\Phi$ and different environments. The growth rate is defined as $\boldsymbol{\Omega} \doteq \boldsymbol{J}_{\star}(\Phi)-\boldsymbol{J}_{\star}(\Phi-1)$, where the balance state $\boldsymbol{J}_{\star}(\Phi)$ is regarded as a function of the averaged connection multiplicity $\Phi$. The growth rate $\Omega$ reflects the localization information contributed by one node.

$\boldsymbol{\Omega}$ converges to a lower value $\boldsymbol{\Lambda}$.

\section{CONCLUding REMARKS}

In this paper, the fundamental limits and spatial cooperation of wireless network localization are studied. It is shown that, the localization accuracy depends on the measurement size, the density of independent reference sources, priori information of node location, the geometric resolution of measurement methodology and the measurement noise intensity. In addition, a remote node can contribute to the localization of another node through spatial propagation of the localization information, if there is a measurement-connection link between them. It is revealed that, the essence of spatial localization cooperation is the spatial propagation of localization information factors. Given a fixed size of network measurements, the node location accuracy will converge statistically to a higher level through spatial localization cooperation, even though the initial locations of the reference nodes are inaccurate. In addition, we have the following conclusions.

- The network localization error propagation principle complies with the Ohm's Law in electric circuit theory, where the measurement accuracy, node location accuracy and measurement-resolution information behave similarly to the resistances connected in parallel or series.

- In a dense network, for the localization of a generic node, the localization information contribution from one of its reference nodes is almost the averaged measurement information $\boldsymbol{\Lambda}$. Hence, the localization accuracy of a node, in cooperation with its $\Phi$ reference nodes, is $\Phi \boldsymbol{\Lambda}$.

- If the measurement size is sufficiently large, the localization accuracy will depend on the priori precision factors only, which is independent of the geometric resolution and measurement methodologies.

Furthermore, a generic balance state of spatial propagation of network localization information is derived in this paper, as well as its upper and lower bounds, which corresponds to the ultimate performance limits of cooperative localization with a fixed size of measurements. The spatial information propagation analysis in this paper can be applied to the TOA, AOA and RSS-based localization.

The spatial information propagation associated with simultaneous localization and tracking will be interesting problems to be investigated in the future.

\section{APPENDIX A}

DERIVATION OF EQ. (20)

Given two squared and invertible matrixes $\mathbf{A}$ and $\mathbf{X}$, the inverse matrix lemma is described as follows,

$$
(\mathbf{A}+\mathbf{X})^{-1}=\mathbf{A}^{-1}-\left(\mathbf{A}^{\top} \mathbf{X}^{-1} \mathbf{A}+\mathbf{A}\right)^{-1}
$$

Based on the above lemma, we have that

$\left(\omega \mathbf{A}_{i, j}+\mathbf{U}_{j}\right)^{-1}=\left(\omega \mathbf{A}_{i, j}\right)^{-1}-\left(\omega^{2} \mathbf{A}_{i, j}^{\top} \mathbf{U}_{j}^{-1} \mathbf{A}_{i, j}+\omega \mathbf{A}_{i, j}\right)^{-1}$

Note that, $\mathbf{A}_{i, j}$ and $\mathbf{U}_{j}$ are symmetric and have full-rank. Hence, $\boldsymbol{\Phi}_{\mathrm{BE}}^{\top}\left(\mathbf{s}_{i}\right)\left(\mathcal{R}_{\mathrm{BE}}\left(\mathbf{s}_{i}\right)\right)^{-1} \boldsymbol{\Phi}_{\mathrm{BE}}\left(\mathbf{s}_{i}\right)$ can be specified as

$$
\begin{aligned}
& \sum_{j \in \Psi_{i}} \omega^{2} \mathbf{A}_{i, j}^{\top}\left(\omega \mathbf{A}_{i, j}+\mathbf{U}_{j}\right)^{-1} \mathbf{A}_{i, j} \\
= & \sum_{j \in \Psi_{i}} \omega \mathbf{A}_{i, j}^{\top}-\sum_{j \in \Psi_{i}} \omega \mathbf{A}_{i, j}^{\top}\left(\omega \mathbf{A}_{i, j}^{\top} \mathbf{U}_{j}^{-1} \mathbf{A}_{i, j}+\mathbf{A}_{i, j}\right)^{-1} \mathbf{A}_{i, j} \\
= & \sum_{j \in \Psi_{i}} \omega \mathbf{A}_{i, j}^{\top}-\sum_{j \in \Psi_{i}}\left(\left(\omega \mathbf{A}_{i, j}\right)^{-1}+\mathbf{U}_{j}^{-1}\right)^{-1} .
\end{aligned}
$$

Hence, the equivalent localization information $\mathcal{I}_{\mathrm{EQ}}\left(\mathbf{s}_{i}\right)$ can be finally expressed as Eq. (20).

\section{APPENDIX B \\ Proof of THEOREM 2}

As indicated in Eq. (22), the current localization information $\mathcal{I}_{\mathrm{EQ}}^{(n)}\left(\mathrm{s}_{i}\right)$ can be read as a function of its last state $\mathcal{I}_{\mathrm{EQ}}^{(n-1)}\left(\mathrm{s}_{i}\right)$, namely, $\mathcal{I}_{\mathrm{EQ}}^{(n)}\left(\mathbf{s}_{i}\right)=f\left(\mathcal{I}_{\mathrm{EQ}}^{(n-1)}\left(\mathbf{s}_{i}\right)\right)$. Based on Eq. (22), when $\mathcal{I}_{\mathrm{EQ}}^{(n-1)}\left(\mathbf{s}_{i}\right) \rightarrow \infty$ and $\mathcal{I}_{\mathrm{EQ}}^{(n-1)}\left(\mathbf{s}_{i}\right) \rightarrow \mathbf{0}$, the localization information of the next step follows Eqs. (46) and (47), respectively. Here, $\mathcal{I}_{\mathrm{EQ}}^{(n-1)}\left(\mathbf{s}_{i}\right) \rightarrow \infty$ means $\mathcal{I}_{\mathrm{EQ}}^{(n-1)}\left(\mathbf{s}_{i}\right)-\mathbf{M} \succeq \mathbf{0}$, $\forall \mathbf{M} \succeq \mathbf{0}$. Moreover, we have $\mathbf{0} \preceq \mathcal{I}_{\mathbf{0}}^{(n)}\left(\mathbf{s}_{i}\right) \preceq \mathcal{I}_{\infty}^{(n)}\left(\mathbf{s}_{i}\right) \prec \infty$. Meanwhile, the SLIP function $\overline{\mathcal{I}}_{\mathrm{EQ}}^{(n)}\left(\mathbf{s}_{i}\right)=f\left(\mathcal{I}_{\mathrm{EQ}}^{(n-1)}\left(\mathbf{s}_{i}\right)\right)$ is monotonously increasing. In brief, the properties of SLIP are summarized as below.

- $\mathcal{I}_{\mathrm{EQ}}^{(n)}\left(\mathbf{s}_{i}\right)=f\left(\mathcal{I}_{\mathrm{EQ}}^{(n-1)}\left(\mathbf{s}_{i}\right)\right)$ is monotonously increasing.

- $\mathbf{0} \preceq \mathcal{I}_{\mathbf{0}}^{(n)}\left(\mathbf{s}_{i}\right) \preceq \mathcal{I}_{\infty}^{(n)}\left(\mathbf{s}_{i}\right) \prec \infty$.

Consequently, there must be one and only one intersection (denoted by $\mathcal{I}_{\mathrm{EQ}}^{\star}\left(\mathbf{s}_{i}\right)$ ) between $\mathcal{I}_{\mathrm{EQ}}^{(n)}\left(\mathbf{s}_{i}\right)=f\left(\mathcal{I}_{\mathrm{EQ}}^{(n-1)}\left(\mathbf{s}_{i}\right)\right)$ and $\mathcal{I}_{\mathrm{EQ}}^{(n)}\left(\mathbf{s}_{i}\right)=\mathcal{I}_{\mathrm{EQ}}^{(n-1)}\left(\mathbf{s}_{i}\right)$, as roughly shown in Fig. 9 .

At the beginning of SLIP (suppose $n=0$ ), since there is no posteriori information about $\mathbf{s}_{i}$, thus $\mathcal{I}_{\mathrm{EQ}}^{(0)}\left(\mathbf{s}_{i}\right)=\mathbf{0}$. Next, with the progress of SLIP $(n=1,2, \cdots)$, the localization information $\mathcal{I}_{\mathrm{EQ}}^{(n)}\left(\mathbf{s}_{i}\right)$ gradually increases and approaches the intersection $\mathcal{I}_{\mathrm{EQ}}^{\star}\left(\mathbf{s}_{i}\right)$ from the left side. Suppose there is a situation that the present localization information $\mathcal{I}_{\mathrm{EQ}}^{(n)}\left(\mathbf{s}_{i}\right)$ grows up such that it exceeds $\mathcal{I}_{\mathrm{EQ}}^{\star}\left(\mathbf{s}_{i}\right)$. Then, at the next propagation step, $\mathcal{I}_{\mathrm{EQ}}^{(n+1)}\left(\mathbf{s}_{i}\right)$ will become lower than $\mathcal{I}_{\mathrm{EQ}}^{(n)}\left(\mathbf{s}_{i}\right)$, since we 


$$
\begin{aligned}
\lim _{\mathcal{I}_{\mathrm{EQ}}^{(n-1)}\left(\mathbf{s}_{i}\right) \rightarrow \infty} \mathcal{I}_{\mathrm{EQ}}^{(n)}\left(\mathbf{s}_{i}\right) & =\sum_{j \in \Psi_{i}}\left(\left(\omega \mathbf{A}_{i, j}\right)^{-1}+\left(\sum_{k \in \Psi_{j} \backslash i} \mathcal{H}_{j, k}^{(n)}+\omega \mathbf{A}_{j, i}+\mathbf{U}_{j}\right)^{-1}\right)^{-1}+\mathbf{U}_{i} \doteq \mathcal{I}_{\infty}^{(n)}\left(\mathbf{s}_{i}\right), \\
\lim _{\mathcal{I}_{\mathrm{EQ}}^{(n-1)}\left(\mathbf{s}_{i}\right) \rightarrow \mathbf{0}} \mathcal{I}_{\mathrm{EQ}}^{(n)}\left(\mathbf{s}_{i}\right) & =\sum_{j \in \Psi_{i}}\left(\left(\omega \mathbf{A}_{i, j}\right)^{-1}+\left(\sum_{k \in \Psi_{j} \backslash i} \mathcal{H}_{j, k}^{(n)}+\mathbf{U}_{j}\right)^{-1}\right)^{-1}+\mathbf{U}_{i} \doteq \mathcal{I}_{\mathbf{0}}^{(n)}\left(\mathbf{s}_{i}\right) .
\end{aligned}
$$

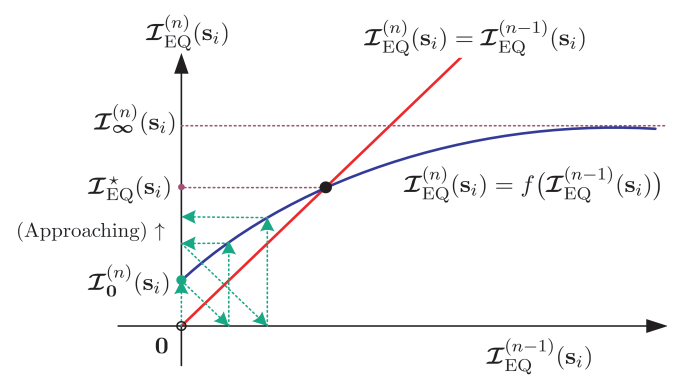

Fig. 9. A rough graph of the SLIP function $\mathcal{I}_{\mathrm{EQ}}^{(n)}\left(\mathbf{s}_{i}\right)=f\left(\mathcal{I}_{\mathrm{EQ}}^{(n-1)}\left(\mathbf{s}_{i}\right)\right)$.

have $\mathcal{I}_{\mathrm{EQ}}^{(n)}\left(\mathbf{s}_{i}\right) \prec \mathcal{I}_{\mathrm{EQ}}^{(n-1)}\left(\mathbf{s}_{i}\right)$ when $\mathcal{I}_{\mathrm{EQ}}^{(n)}\left(\mathbf{s}_{i}\right) \succ \mathcal{I}_{\mathrm{EQ}}^{\star}\left(\mathbf{s}_{i}\right)$, according to SLIP properties, as also shown in Fig. 9.

In a word, the localization information will gradually level up to $\mathcal{I}_{\mathrm{EQ}}^{\star}\left(\mathbf{s}_{i}\right)$, and then keep balance until there is more measurement input. Hence Theorem 2 is proved.

\section{APPENDIX C \\ DERIVATION OF EQ. (29)}

Since the equation $\left(\boldsymbol{\Lambda}^{-1}+\boldsymbol{J}_{\star}^{-1}\right)^{-1}=\boldsymbol{J}_{\star}\left(\boldsymbol{\Lambda}+\boldsymbol{J}_{\star}\right)^{-1} \boldsymbol{\Lambda}$ holds, Eq. (28) can be equivalently expressed as below

$$
\begin{aligned}
\boldsymbol{J}_{\star} & =\Phi \boldsymbol{J}_{\star}\left(\boldsymbol{\Lambda}+\boldsymbol{J}_{\star}\right)^{-1} \boldsymbol{\Lambda}+\mathbf{U}, \\
\boldsymbol{J}_{\star} \boldsymbol{\Lambda}^{-1}\left(\boldsymbol{\Lambda}+\boldsymbol{J}_{\star}\right) & =\Phi \boldsymbol{J}_{\star}+\mathbf{U} \boldsymbol{\Lambda}^{-1}\left(\boldsymbol{\Lambda}+\boldsymbol{J}_{\star}\right), \\
\boldsymbol{J}_{\star} \boldsymbol{\Lambda}^{-1} \boldsymbol{J}_{\star}+\boldsymbol{J}_{\star} & =\Phi \boldsymbol{J}_{\star}+\mathbf{U}+\mathbf{U} \boldsymbol{\Lambda}^{-1} \boldsymbol{J}_{\star} .
\end{aligned}
$$

Hence, the balance equation can be further cast as

$$
\boldsymbol{J}_{\star} \boldsymbol{\Lambda}^{-1} \boldsymbol{J}_{\star}-\mathbf{U}(\underbrace{(\Phi-1) \mathbf{U}^{-1}+\boldsymbol{\Lambda}^{-1}}_{\mathrm{G}}) \boldsymbol{J}_{\star}-\mathbf{U}=\mathbf{0} .
$$

Consequently, Eq. (29) is obtained.

\section{APPENDIX D}

DERIVATION OF EQ. (31)

At first, we give two conclusions below, which are useful for deriving the balance state $\boldsymbol{J}_{\star}$.

$$
\begin{aligned}
\mathbf{U G} \boldsymbol{J}_{\star} & =\boldsymbol{J}_{\star} \mathbf{G} \mathbf{U}, \\
\boldsymbol{\Lambda}^{-\frac{1}{2}} \mathbf{U G} \boldsymbol{\Lambda}^{\frac{1}{2}} & =\boldsymbol{\Lambda}^{\frac{1}{2}} \mathbf{G} \mathbf{U} \boldsymbol{\Lambda}^{-\frac{1}{2}} .
\end{aligned}
$$

Based on the fact $\mathbf{G}=(\Phi-1) \mathbf{U}^{-1}+\boldsymbol{\Lambda}^{-1}$, Eq. (53) can be directly proved. The balance state $\boldsymbol{J}_{\star}$ meets with Eq. (52), which will be proved in APPENDIX E.

Consequently, based on Eq. (29), we have Eqs. (54)-(58), where Eqs. (55) and (57) have used the results shown in Eqs. (52) and (53), respectively. Moreover, the balance equation can be further derived as

$$
\begin{aligned}
\boldsymbol{\Lambda}^{-\frac{1}{2}} \boldsymbol{J}_{\star} \boldsymbol{\Lambda}^{-\frac{1}{2}}= & \frac{1}{2}\left(4 \boldsymbol{\Lambda}^{-\frac{1}{2}} \mathbf{U} \boldsymbol{\Lambda}^{-\frac{1}{2}}+\boldsymbol{\Lambda}^{-\frac{1}{2}} \mathbf{U G} \boldsymbol{\Lambda} \mathbf{G} \mathbf{U} \boldsymbol{\Lambda}^{-\frac{1}{2}}\right)^{\frac{1}{2}} \\
& +\frac{1}{2} \boldsymbol{\Lambda}^{-\frac{1}{2}} \mathbf{U G} \boldsymbol{\Lambda}^{\frac{1}{2}}
\end{aligned}
$$

By pre-multiplying and post-multiplying $\boldsymbol{\Lambda}^{\frac{1}{2}}$ at both sides of Eq. (59), the balance state in Eq. (31) is thus obtained.

\section{APPENDIX E}

DERIVATION OF EQ. (52)

Since $\left(\boldsymbol{\Lambda}^{-1}+\boldsymbol{J}_{\star}^{-1}\right)^{-1}=\boldsymbol{\Lambda}\left(\boldsymbol{\Lambda}+\boldsymbol{J}_{\star}\right)^{-1} \boldsymbol{J}_{\star}$ also holds, Eq. (28) can be rewritten as

$$
\boldsymbol{J}_{\star}=\Phi \boldsymbol{\Lambda}\left(\boldsymbol{\Lambda}+\boldsymbol{J}_{\star}\right)^{-1} \boldsymbol{J}_{\star}+\mathbf{U} .
$$

By doing similar manipulations with Eqs. (49) and (50), Eq. (28) can also be expressed as

$$
\boldsymbol{J}_{\star} \boldsymbol{\Lambda}^{-1} \boldsymbol{J}_{\star}-\boldsymbol{J}_{\star} \mathbf{G} \mathbf{U}-\mathbf{U}=\mathbf{0} .
$$

Combing with Eq. (51), we can see that, the balance state meets with Eq. (52), namely, $\mathbf{U G} \boldsymbol{J}_{\star}=\boldsymbol{J}_{\star} \mathbf{G U}$.

\section{REFERENCES}

[1] M. D. Dikaiakos, A. Florides, T. Nadeem and L. Iftode, "Location-aware services over vehicular ad-hoc networks using car-to-car communication," IEEE Journal on Selected Areas in Communications, 25.8 (2007): 15901602.

[2] Y. Cao, Z. Sun, N. Wang and M. Riaz, "Geographic-Based Sprayand-Relay (GSaR): An Efficient Routing Scheme for DTNs." IEEE Transactions on Vehicular Technology 64.4(2015):1548-1564.

[3] Y. Cao, H. Cruickshank and Z. Sun, "A routing framework for Delay Tolerant Networks based on encounter angle," Wireless Communications and Mobile Computing Conference (IWCMC), 2011 7th International 2231 - 2236, 4-8 July 2011.

[4] E. Kaasinen, "User needs for location-aware mobile services," Personal and ubiquitous computing 7.1 (2003): 70-79.

[5] M. Li, H. Zhu, Z. Gao, S. Chen, L. Yu, S. Hu and K. Ren, "All your location are belong to us: breaking mobile social networks for automated user location tracking," MobiHoc 2014: 43-52

[6] H. Li, L. Sun, Haojin Zhu, X. Lu and X. Cheng, "Achieving privacy preservation in WiFi fingerprint-based localization". INFOCOM, 2014 Proceedings IEEE, vol., no., pp.2337-2345, April 27-May 22014

[7] S. M. Kay, Fundamentals of Statistical Signal Processing, Volume 2: Detection theory. Prentice Hall PTR, 1998.

[8] P. Lamon, I. Nourbakhsh, B. Jensen and R. Siegwart, "Deriving and matching image fingerprint sequences for mobile robot localization." ICRA. May, 2001: pp. 1609-1614.

[9] G. Schroth, R. Huitl, D. Chen, M. Abu-Alqumsan, A. Al-Nuaimi and E. Steinbach, "Mobile visual location recognition," IEEE Signal Processing Magazine, vol. 28, no. 4, pp. 77C89, July 2011.

[10] X. Sheng and Y. Hu. "Maximum likelihood multiple-source localization using acoustic energy measurements with wireless sensor networks." Signal Processing, IEEE Transactions on 53.1 (2005): 44-53.

[11] G. Sun, J. Chen, W. Guo, and K. Liu, Signal processing techniques in network-aided positioning: a survey of state-of-the-art positioning designs, IEEE Signal Processing Magazine, vol. 22, no. 4, pp. 12C23, July 2005. 


$$
\begin{aligned}
\boldsymbol{J}_{\star} \boldsymbol{\Lambda}^{-1} \boldsymbol{J}_{\star}-\mathbf{U G} \boldsymbol{J}_{\star} & =\mathbf{U}, \\
\left(\boldsymbol{J}_{\star} \boldsymbol{\Lambda}^{-\frac{1}{2}}-\frac{1}{2} \mathbf{U G} \boldsymbol{\Lambda}^{\frac{1}{2}}\right)\left(\boldsymbol{\Lambda}^{-\frac{1}{2}} \boldsymbol{J}_{\star}-\frac{1}{2} \boldsymbol{\Lambda}^{\frac{1}{2}} \mathbf{G U}\right) & =\mathbf{U}+\left.\frac{1}{4} \mathbf{U G} \boldsymbol{\Lambda} \mathbf{G U}\right|_{\text {employing Eq. (52) }}, \\
\left(\boldsymbol{\Lambda}^{-\frac{1}{2}} \boldsymbol{J}_{\star} \boldsymbol{\Lambda}^{-\frac{1}{2}}-\frac{1}{2} \boldsymbol{\Lambda}^{-\frac{1}{2}} \mathbf{U G} \boldsymbol{\Lambda}^{\frac{1}{2}}\right)\left(\boldsymbol{\Lambda}^{-\frac{1}{2}} \boldsymbol{J}_{\star} \boldsymbol{\Lambda}^{-\frac{1}{2}}-\frac{1}{2} \boldsymbol{\Lambda}^{\frac{1}{2}} \mathbf{G} \mathbf{U} \boldsymbol{\Lambda}^{-\frac{1}{2}}\right) & =\boldsymbol{\Lambda}^{-\frac{1}{2}} \mathbf{U} \boldsymbol{\Lambda}^{-\frac{1}{2}}+\frac{1}{4} \boldsymbol{\Lambda}^{-\frac{1}{2}} \mathbf{U G} \boldsymbol{\Lambda} \mathbf{G} \mathbf{U} \boldsymbol{\Lambda}^{-\frac{1}{2}} \\
\left(\boldsymbol{\Lambda}^{-\frac{1}{2}} \boldsymbol{J}_{\star} \boldsymbol{\Lambda}^{-\frac{1}{2}}-\frac{1}{2} \boldsymbol{\Lambda}^{-\frac{1}{2}} \mathbf{U G} \boldsymbol{\Lambda}^{\frac{1}{2}}\right)^{2} & =\boldsymbol{\Lambda}^{-\frac{1}{2}} \mathbf{U} \boldsymbol{\Lambda}^{-\frac{1}{2}}+\left.\frac{1}{4} \boldsymbol{\Lambda}^{-\frac{1}{2}} \mathbf{U G} \boldsymbol{\Lambda} \mathbf{G} \mathbf{U} \boldsymbol{\Lambda}^{-\frac{1}{2}}\right|_{\text {using }(53)}, \\
\boldsymbol{\Lambda}^{-\frac{1}{2}} \boldsymbol{J}_{\star} \boldsymbol{\Lambda}^{-\frac{1}{2}}-\frac{1}{2} \boldsymbol{\Lambda}^{-\frac{1}{2}} \mathbf{U G} \boldsymbol{\Lambda}^{\frac{1}{2}} & =\left(\boldsymbol{\Lambda}^{-\frac{1}{2}} \mathbf{U} \boldsymbol{\Lambda}^{-\frac{1}{2}}+\frac{1}{4} \boldsymbol{\Lambda}^{-\frac{1}{2}} \mathbf{U G} \boldsymbol{\Lambda} \mathbf{G} \mathbf{U} \boldsymbol{\Lambda}^{-\frac{1}{2}}\right)^{\frac{1}{2}}
\end{aligned}
$$

[12] Z. Yang, Z. Zhou and Y. Liu, "From RSSI to CSI: Indoor localization via channel response." ACM Computing Surveys (CSUR) 46.2 (2013): 25.

[13] S. Jung, S. Hann and C. Park. "TDOA-based optical wireless indoor localization using LED ceiling lamps." Consumer Electronics, IEEE Transactions on 57.4 (2011): 1592-1597.

[14] Y. Shen and M. Z. Win, "Fundamental limits of wideband localizationPart I: A General Framework," IEEE Trans. Inf. Theory, vol.56, no.10, 2010, pp.4956-4979.

[15] Y. Shen, H. Wymeersch and M. Z. Win, "Fundamental limits of wideband localization-Part II: Cooperative Networks," IEEE Trans. Inf. Theory, vol.56, no.10, 2010, pp.4956-4979.

[16] M. Z. Win, A. Conti, S. Mazuelas, Y. Shen, W. M. Gifford, D. Dardari and M. Chiani, "Network localization and navigation via cooperation," IEEE Communications Magazine 49.5 (2011): 56-62.

[17] J. Teng, H. Snoussi, C. Richard and R. Zhou, "Distributed Variational Filtering for Simultaneous Sensor Localization and Target Tracking in Wireless Sensor Networks," IEEE Trans. Veh. Techn., vol.61, no.5, 2012, pp.2305-2318.

[18] B. Hao, "On the Cramer-Rao Bound of Multiple Sources Localization Using RDOAs and GROAs in the Presence of Sensor Location Uncertainties," Proc. IEEE WCNC, 2012.

[19] S. Mazuelas, Y. Shen, and M. Z. Win, "Information coupling in cooperative localization," Communications Letters, IEEE 15.7 (2011): 737-739.

[20] Y. Shen, S. Mazuelas, and M. Z. Win, "Network navigation: Theory and interpretation," IEEE Journal on Selected Areas in Communications 30.9 (2012): 1823-1834.

[21] F. Gustafsson and F. Gunnarsson, "Mobile positioning using wireless networks: possibilities and fundamental limitations based on available wireless network measurements," IEEE Signal Processing Magazine 22.4 (2005): 41-53.

[22] M. Angjelichinoski, D. Denkovski, V. Atanasovski, L. Gavrilovska, "Cramer-Rao Lower Bounds of RSS-Based Localization With Anchor Position Uncertainty," IEEE Trans. Inf. Theory 61.5 (2015): 2807-2834.

[23] I. Sharp, K. Yu and T. Sathyan, "Positional accuracy measurement and error modeling for mobile tracking," Mobile Computing, IEEE Transactions on 11.6 (2012): 1021-1032.

[24] I. Güvenç, C. C. Chong, F. Watanabe and H. Inamura, "NLOS identification and weighted least-squares localization for UWB systems using multipath channel statistics," EURASIP Journal on Advances in Signal Processing 2008 (2008): 36.

[25] A. Conti, M. Guerra, D. Dardari, N. Decarli and M. Z. Win, "Network experimentation for cooperative localization," IEEE Journal on Selected Areas in Communications, Vol.30, No.2, 2012, pp.467-475.

[26] I. Güvenç and C. C. Chong. "A survey on TOA based wireless localization and NLOS mitigation techniques," Communications Surveys \& Tutorials, IEEE 11.3 (2009): 107-124.

[27] G. Giorgetti, S. K. Gupta, and G. Manes, ”Understanding the limits of RF-based collaborative localization," Networking, IEEE/ACM Transactions on 19.6 (2011): 1638-1651.

[28] R. W. Ouyang, A.K-S. Wong, and C.-T. Lea, "Received Signal Strengthbased Wireless Localization via Semidefinite Programming: Noncooperative and Cooperative schemes," IEEE Trans. Veh. Techn., vol.59, no.3, 2010, pp.1307-1318

[29] P. Rong and M. L. Sichitiu, "Angle of arrival localization for wireless sensor networks," Proc. of 2006 3rd Annual IEEE Communications Society on Sensor and Ad Hoc Communications and Networks (SECON'06), 2006.

[30] J. Xu, M. Ma and C. L. Law, "Cooperative angle-of-arrival position localization," Measurement, Vol.59, 2015, pp.302-313.
[31] P. M. Djuric, M. V. Vemula, M. R. Bugallo and J. Miguez, "Noncooperative Localization of Binary Sensors." Proc. IEEE 13th Workshop on Statistical Signal Processing, 2005.

[32] Y. Dodge, "The Oxford dictionary of statistical terms." Oxford University Press (2003)

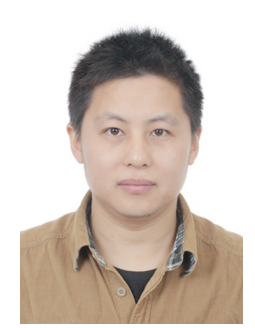

Bingpeng Zhou received the B.E. degree in electronic \& information engineering from Zhongyuan University of Technology, China, in 2010. He is currently working toward the Ph.D. degree with the School of Information Science and Technology, Southwest Jiaotong University, Chengdu, China. He was a Visiting Ph.D. Student at the 5G Innovation Centre, University of Surrey, Guildford, U.K., from September to November, 2015. His current research interests include wireless localization \& tracking, distributed Bayesian inference \& filtering, machine learning, and wireless fast-time-varying communication systems.

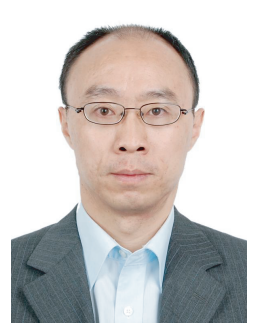

Qingchun Chen (M'06-SM'14) received the B.Sc and M.Sc degree (with Hons.) from Chongqing University, Chongqing, China, in 1994 and 1997, respectively. and the Ph.D. degree from Southwest Jiaotong University, Chengdu, China, in 2004. He has been with Southwest Jiaotong University, as an Associate Professor, since 2004 and then as a Full Professor since 2009. He has authored and coauthored more than 80 research papers, 2 book chapters and 20 patents. Currently, he is an Associate Editor of the IEEE ACCESS JOURNAL. His research interests include wireless communication, wireless network, channel coding, and signal processing.

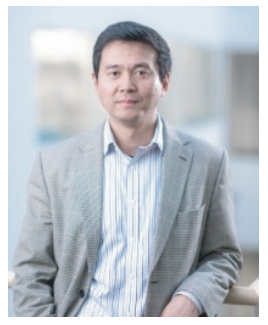

Pei Xiao (SM'11) received the $\mathrm{PhD}$ degree from Chalmers University of Technology, Sweden in 2004. Prior to joining the University of Surrey in 2011, he worked as a research fellow at Queen's University Belfast and had held positions at Nokia Networks in Finland. He is a Reader at University of Surrey and also the technical manager of 5G Innovation Centre (5GIC), leading and coordinating research activities in all the work areas in 5GIC (http://www.surrey.ac.uk/5gic/what-5g). Dr. Xiao's research interests and expertise span a wide range of areas in communications theory and signal processing for wireless communications.

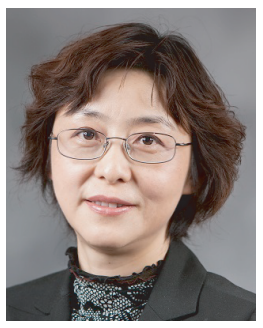

Lian Zhao (S'99-M'03-SM'06) received the Ph.D. degree from the University of Waterloo, Waterloo, ON, Canada, in 2002. Since 2003, she has been with the Department of Electrical and Computer Engineering (ELCE), Ryerson University, Toronto, ON, first as an Assistant Professor, then as an Associate Professor (2007), and currently as a Professor. Since 2013, she has been the Program Director for Graduate Studies with ELCE, Ryerson University. Her research interests include wireless communications, radio resource management, power control, cognitive radio and cooperative communications, and design and applications of the energy-efficient wireless sensor networks. 
Dr. Zhao has served as the Symposium Cochair for the 2013 IEEE GLOBal Communications Conference Communication Theory SympoSIUM, the Web Conference Cochair for the 2009 IEEE TORONTO INTERNATiOnal CONFERENCE SCIENCE AND TeChNOlogy FOR Humanity, the IEEE Ryerson Student Branch Counselor since 2005, and a technical program committee member for numerous IEEE flagship conferences.

She served as a Guest Editor for the INTERNATIONAL JOURNAL ON Communication Networks and Distributed Systems, Special Issue on Cognitive Radio Networks in 2011, as an Associate Editor for the IEEE TRANSACTIONS ON Vehicular TECHNOLOGY since 2013, and as a Reviewer for IEEE TRANSACTIONS as well as for various Natural Sciences and Engineering Research Council proposals. She received the Canada Foundation for Innovation New Opportunity Research Award in 2005; the Ryerson Faculty Merit Award in 2005 and 2007; the Faculty Research Excellence Award from ELCE, Ryerson University, in 2010 and 2012; the Best Student Paper Award (with her student) from Chinacom in 2011; and the Best Paper Award (with her student) from the 2013 International Conference on Wireless Communications and Signal Processing. She is a licensed Professional Engineer in Ontario and a member of the IEEE Communication/Vehicular Society. 